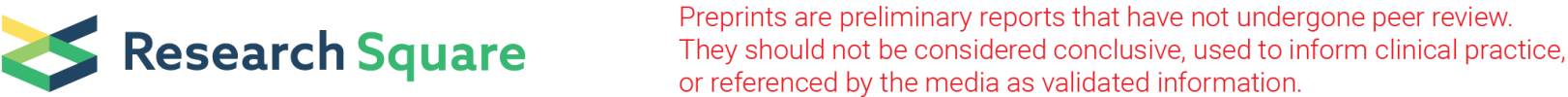

\section{Prevalence of Treponema species in the Gut Microbiome is Linked to Bifidobacterium sp. and Bacteroides sp.}

\section{Souad BELKACEMI}

Aix-Marseille Université IHU Méditerranée Infection: IHU Mediterranee Infection

\section{MARYAM TIDJANI ALOU}

Aix-Marseille Université IHU Méditerranée Infection: IHU Mediterranee Infection

\section{Matthieu MILLION}

Aix-Marseille Université IHU Méditerranée Infection: IHU Mediterranee Infection

Anthony LEVASSEUR

Aix-Marseille Université IHU Méditerranée Infection: IHU Mediterranee Infection

\section{Saber KHELAIFIA}

Aix-Marseille Université IHU Méditerranée Infection: IHU Mediterranee Infection

Didier RAOULT ( $\square$ didier.raoult@gmail.com )

Aix-Marseille Universite https://orcid.org/0000-0003-0771-8245

\section{Research}

Keywords: Human microbiota, Treponema, Bifidobacterium, metagenomics, culture, inhibition, antagonism, growth

Posted Date: December 4th, 2020

DOI: https://doi.org/10.21203/rs.3.rs-117420/v1

License: (c) (1) This work is licensed under a Creative Commons Attribution 4.0 International License. Read Full License 


\section{Abstract}

\section{Background}

Treponema species as commensals of the human microbiome have a prevalence and function depending on the studied niche. In the oral cavity, treponemes are ubiquitous while they have been strictly linked to rural and ancestral populations in the gastro-intestinal tract and are undetected in urban populations. In this study, an in silico analysis of 1481 metagenomes, selected based on the previous detection of treponemes in such sample types, was conducted to predict putative bacterial antagonists of treponemes, i.e. bacteria present when treponemes are lacking and vice versa. Predicted antagonists were subsequently tested in-vitro against Treponema denticola CSUR P7640.

\section{Results}

Most frequent predicted antagonists included members of the Bifidobacteriaceae family $(23.3 \%)$ among which Bifidobacterium longum was the most significant (63.3\% in T- group, $p<0.0000001$ ) as well as members of the Bacteroides (6.7\%) and Streptococcus (13.3\%) genera. The relative abundance of the aforementioned taxa was also anticorrelated with that of the Treponema genus in the metagenomes analyzed in this study. B. Iongum CSUR P7400, Bifidobacterium bifidum CSUR P1194, Bifidobacterium breve CSUR P7882, Bacteroides ovatus CSUR P4577, Bacteroides uniformis CSUR P2248 and Bacteroides thetaiotaomicron CSUR P7324 were able to inhibit the growth of $T$. denticola with B. longum being the most efficient.

\section{Conclusions}

These results highlight an antagonism between Bifidobacterium species, known probiotics and biopreservatives, and Treponema spp. This may help to explain the variation of Treponema prevalence in high-income countries and middle- and low-income countries which might linked to the differential consumption of fermented dairy foodstuff and processed food and therefore to the phenomenon of nutrition transition.

\section{Background}

Lifestyle has a major impact on the composition of the gut microbiome [1, 2]. It has been showed that urban populations have a different composition of the gut microbiome than that of rural populations [36]. In fact, diet shifts the composition of the gut microbiota towards specific profiles according to the dominant macronutrient in the diet [7]. One example of a discriminating taxon between urban and rural populations are Treponema spp. These fastidious species have only been isolated from the oral cavity in humans [8] but have been detected in the human gut using molecular methods [6]. The Treponema genus has been described in the literature as strictly associated with rural populations and completely lost in urban populations $[6,9,10]$. Several studies have shown a relative abundance in Treponema spp., specifically in the gut of rural populations (Native Americans from Venezuela, Hadza hunter-gatherers 
from Tanzania, Bedouin, from Saudi Arabia, Matses and Tunapuco populations from Peru, Pygmies from Cameroon and Congo, Amazonians from French Guyana, populations from rural Burkina faso and rural Malawi) in opposition to urban populations from several countries such as France including French Guyana and French Polynesia, Italy, USA, Cameroon, Nigeria, Senegal and Saudi Arabia [2-5, 11-14]. Moreover, the ancestral human microbiome is seemingly similar to that of rural populations with a shared characteristic, the prevalence in treponemes [15].

Several hypotheses have been suggested to explain this divergence in urban and rural areas such as a diet rich in fiber and complex polysaccharides, the use of antimicrobial compounds, the consumption of fermented milk or a possible transmission from animals to humans $[6,12,14]$. However, the mechanisms underlying this phenomenon are still unknown. In this study, we aim to explore the flora associated with the presence or absence of treponemes in the human microbiota through in silico analyses to determine natural antagonists and predictors of treponemes and confirm in vitro the predicted microbe-microbe interactions.

\section{Methods}

\section{Samples}

In this study, 1481 human samples were analyzed, collected from individuals with different health status and geographical origins (Table 1). The sample selection was based on the possible prevalence of Treponema in their microbiota according to the literature. 251 urine samples, 29 saliva samples and 1201 stool samples were therefore selected. Ethical approval was obtained for this study from the ethics committee of "IHU Méditerranée Infection" under number 2016-011 (Marseille, France). 
Table 1

Analyzed metagenomes and their samples of origins (niche and geographic location).

\begin{tabular}{|c|c|c|c|c|c|c|c|}
\hline & & $T+$ & & & $\mathrm{T}-$ & & \\
\hline Country & Population & Feces & $\begin{array}{l}\text { Oral } \\
\text { cavity }\end{array}$ & Urine & Feces & $\begin{array}{l}\text { Oral } \\
\text { cavity }\end{array}$ & Urine \\
\hline \multirow[t]{2}{*}{ France } & & 0 & 22 & 5 & 214 & 7 & 246 \\
\hline & $\begin{array}{l}\text { Amazonian (French } \\
\text { Guyana) }\end{array}$ & 24 & 0 & 0 & 27 & 0 & 0 \\
\hline Congo & Pygmies & 42 & 0 & 0 & 85 & 0 & 0 \\
\hline $\begin{array}{l}\text { Saudi } \\
\text { Arabia }\end{array}$ & Bedouins & 5 & 0 & 0 & 20 & 0 & 0 \\
\hline \multirow[t]{3}{*}{ Senegal } & Dielmo & 12 & 0 & 0 & 10 & 0 & 0 \\
\hline & Ndiop & 17 & 0 & 0 & 21 & 0 & 0 \\
\hline & Dakar & 0 & 0 & 0 & 68 & 0 & 0 \\
\hline \multirow[t]{2}{*}{ Mali } & Bandiagara & 21 & 0 & 0 & 188 & 0 & 0 \\
\hline & Koulikoro & 3 & 0 & 0 & 403 & 0 & 0 \\
\hline Niger & Niamey & 0 & 0 & 0 & 41 & 0 & 0 \\
\hline All & & 124 & 22 & 5 & 1077 & 7 & 246 \\
\hline
\end{tabular}

\section{In silico search of antagonistic bacteria of Treponema}

\section{Genomic DNA sequencing}

DNA from 1481 samples was extracted using the NucleoSpin ${ }^{\circledR}$ Tissue Mini kit (Macherey Nagel, Hoerdt, France), after a protease step by incubation at $37^{\circ} \mathrm{C}$ for $15 \mathrm{~min}$ with Proteinase K (Macherey Nagel, Hoerdt, France) and a deglycosylation step by incubation at $37^{\circ} \mathrm{C}$ overnight (Biolabs, England). The extracted DNA was then amplified and 16S rRNA was sequenced using the MiSeq technology (Illumina, Inc., SanDiego CA 92121, USA). For each extraction protocol, DNA was amplified for the 16S rRNA "V3-V4" regions by PCR for 40 cycles, using Kapa HiFi Hotstart ReadyMix 2x primers (Kapa Biosystems Inc., Wilmington, MA, USA) and the surrounding conserved region V3_V4 primers with overhang adapters: (FwOvAd_341FTCGTCGGCAGCGTCAGATGTGTATAAGAGACAGCCTACGGGGGGGGCGCGC; RevOvAd_785R GTCTCGTGGGCTCGGAGAGGGGATATAGAGDAGGAGATA [9]. The paired end reads of the 
corresponding raw fastq files were assembled into contigs using Pandaseq [32]. The high-quality sequences were then selected for the next steps of analysis by considering only the sequences containing both primers (forward and reverse). In the following filtering steps, the sequences containing $\mathrm{N}$ were removed. Sequences with length shorter than $200 \mathrm{nt}$ were also removed, and sequences longer than 500 nt were trimmed. Both forward and reverse primers were also removed from each of the sequences. An additional filtering step was applied to remove the chimeric sequences using UCHIME [33] of USEARCH [34]. The filtering steps were performed using the QIIME pipeline [35]. Strict dereplication (clustering of duplicate sequences) was performed on the filtered sequences, and they were then sorted by decreasing number of abundance [36-38]. For each metagenome, the clustering of OTUs was performed with $97 \%$ identity.

\section{Data processing}

The resulting OTUs were assigned using MetaGX, a home-made software pipeline developed with the start-up XeGen (http://xegen.eu/) based on QIIME [35] and using BLAST [39] for taxonomic assignment and SILVA $[40,41]$ as a reference dictionary (See the supplemental material). For taxonomic assignments, we applied at least 20 reads per OTU. The OTUs were then searched against each database using BLASTN [39]. The best match of $\geq 97 \%$ identity and $100 \%$ coverage for each of the OTUs was extracted from the reference database, and taxonomy was assigned up to the species level.

\section{Methodology}

From a total of 2668 metagenomes in MetaGX V2D2, 1481 metagenomes were selected and separated into two groups: a group of 1330 Treponema-negative metagenomes ( $\mathrm{T}-$ ) among which 246 urine samples, 7 saliva samples, and $1077 \mathrm{fecal}$ samples and a group of 151 Treponema-positive metagenomes $(T+)$ containing 5 urine samples, 22 saliva samples, and 124 fecal samples (Figure 1). We sought to identify the most enriched or depleted species using frequency difference (frequency in $\mathrm{T}+$ samples versus frequency in T- samples). Frequency analysis has the advantage compared to abundance analysis to not be subjected to depth bias and thus not require a method of normalization [42]. Antagonistic species were the most frequently detected species in the T- group with a high statistical significance. Conversely, predictors were the most frequently detected species (top 30 species) in the T+ group with a high statistical significance.

\section{Statistical analysis}

The data were entered into the Microsoft Office Excel template, then imported and analyzed in the statistical software SPSS, version 20 (IBM Corp., Armonk, NY) using bilateral uncorrected chi-square test $(\mathrm{X})$, with a significance level at $\mathrm{P} \leq 0.05$. 


\section{In vitro proof-of concept of antagonistic activity against Treponema sp.}

In order to confirm the predicted antagonists detected in silico, we performed in vitro tests using the antibiotic discovery method [43]. This method reveals antibacterial effects, based on the principle that a bacterium inoculated into a solid medium [8] or a substance produced this bacterium diffusing into the solid culture medium can inhibit a target strain subsequently inoculated onto the medium.

In this work we used Treponema denticola CSURP 7640, isolated in our laboratory from the oral cavity against which top ranking predicted antagonists were tested.

Tests were carried out in an anaerobic chamber (Don Whitley Scientific Limited, West Yorkshire, UK) under an atmosphere composed of $80 \% \mathrm{~N} 2,15 \% \mathrm{CO} 2$ and $5 \% \mathrm{H} 2$, using T-Raoult culture medium [8], supplemented with $8 \mathrm{~g} / \mathrm{L}$ of agar (Fisher Scientific, Illkirch, France). The growth of antagonistic bacteria recovered from the rickettsia unit strain collection (CSUR) was previously tested on T-Raoult medium. A 0.5 McFarland suspension was prepared for each antagonist identified. In order to obtain a homogeneous growth of treponemes inside the solid medium, different concentrations were tested, namely $0.5,1,1.5$ and $2 \mathrm{McF}$ arland, the latter being the optimum concentration. Treponema inoculation was performed inside the agar as described previously [8]. $5 \mathrm{~mm}$-diameter wells were made using a sterile punch (Biosegama, Brumath, France) on each solidified agar inoculated with T. denticola. Each well was filled with $50 \mu \mathrm{L}$ of a suspension at $0.5 \mathrm{McF}$ of the selected antagonists. The cultures are then incubated in the anaerobic chamber at $37^{\circ} \mathrm{C}$. After a minimal incubation period of 4 days, the diameters of the inhibition zones appearing around the wells were measured using Scan 1200 (LABO-MODERNE, Gennevilliers, France). Three replicates were performed for each test with PBS as a negative control in each test. Control plates inoculated or not with T. denticola (Figure 7A and 6B) were incubated in the same conditions as a growth control. A positive control was also carried out using a vancomycin disc

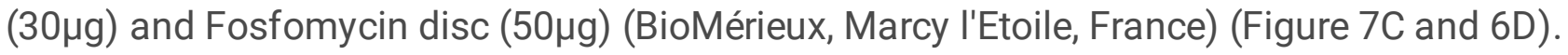

\section{Results}

\section{Top ranking predicted antagonists}

The most common bacterial taxa among predicted antagonists belonged to the Bifidobacteriaceae family (23.3\%) followed by the Streptococcus (13.3\%) and Bacteroides (6.7\%) genera (Figure 2). The relative abundance of these predicted antagonists according to that of Treponema spp. showed a significant anticorrelation (Figure 3 ) between Bifidobacterium spp. $\left(r^{2}=-0.2016, p<0.0001\right)$, Streptococcus spp. $\left(r^{2}=-0.2557, p<0.0001\right)$ and Bacteroides spp. $\left(r^{2}=-0.04911, p=0.05\right)$ (Figures 3A, 3B and 3C respectively).

The OTUs belonging to the Bifidobacteriaceae family were the following: Bifidobacterium longum subsp.infantis / Bifidobacterium longum subsp.longum / Bifidobacterium longum subsp. suillum (63.3\% in T- group, $p<0.0000001)$, Bifidobacterium adolescentis / Bifidobacterium catenulatum / 
Bifidobacterium faecale / Bifidobacterium kashiwanohense / Bifidobacterium pseudocatenulatum / Bifidobacterium ruminantium (36.9\% in T- group, $p<0.0000001)$, Bifidobacterium bifidum $(25.9 \%$ in Tgroup, $p<0.0000001)$, Bifidobacterium breve (21.9\% in T- group, $p<0.0000001)$, IHU PS 91 Bifidobacteriaceae $1548\left(22.9 \%, p=3,67 \mathrm{E}^{-06}\right)$ and IHU PS 92 Bifidobacteriaceae 289 (14.1\% in T- group, $p=$ $\left.9,69 \mathrm{E}^{-05}\right)$, Bifidobacterium adolescentis / Bifidobacterium faecale / Bifidobacterium ruminantium (35.1\% in T- group, $p=4,74 \mathrm{E}^{-03}$ ). The OTUs belonging to the Streptococcus genus consisted of Streptococcus salivarius subsp. salivarius / Streptococcus salivarius subsp. thermophilus / Streptococcus vestibularis (37.2\% in T- group, $p<0.0000001$ ), Streptococcus ihumii / Streptococcus lactarius / Streptococcus peroris / Streptococcus sinensis (27.6\% in T- group, $p<0.0000001)$, Streptococcus alactolyticus / Streptococcus equinus / Streptococcus gallolyticus subsp. macedonicus / Streptococcus gallolyticus subsp. pasteurianus / Streptococcus infantarius subsp. coli / Streptococcus infantarius subsp. infantarius / Streptococcus lutetiensis (28.6\% in T- group, $p<0.0000001)$ and IHU PS 96 Streptococcus 56258 (11.5\% in T- group, $p=5,27 \mathrm{E}^{-07}$ ) (Figure 2). As for the Bacteroides genus, the OTUs were assigned to Bacteroides fragilis (29.6\% in T- group, $\left.p=9,02 \mathrm{E}^{-04}\right)$ and Bacteroides thetaiotaomicron (19.9\% in T- group, $p=3,67 \mathrm{E}^{-}$ $03)$.

When analyzing the metagenomes within sample type, fecal samples followed the same trend previously observed namely a high representation of Bifidobacteriaceae (23.3\%) and Streptococcus (13\%) (Figure 4B) whereas these taxa were not represented among top ranking antagonists. In fact, the most common predicted antagonists in oral samples included taxa belonging to the Prevotellaceae (23.3\%), Veillonellaceae (20\%) and Actinomycetaceae (13.3\%) families (Figure 4D). To exclude lifestyle as the sole selective pressure, we also analyzed rural metagenomes according to the prevalence of treponemes. Among the 263 fecal metagenomes selected (51 Amazonians, 25 Bedouins, 127 pygmies, 60 Senegalese), 163 metagenomes (27 Amazonians, 20 Bedouins, 85 pygmies, 31 Senegalese) belonged to the T- group while the T+ group consisted of 100 metagenomes (24 Amazonians, 5 Bedouins, 42 pygmies, 29 Senegalese). Bacteroides (16.7\%) and Bifidobacterium (10\%) were the most frequent OTUs among predicted antagonists (Figure 5B).

\section{Bacteria associated to the prevalence in Treponema spp.}

The top 30 OTUs in T+ samples belonged to the Clostridiales order (33.3\%) and more specifically the Ruminococcaceae (13.3\%), Eubacteriaceae (10\%) and Lachnospiraceae (6.7\%) families. OTUs belonging to the Ruminococcaceae family consisted of: Colidextribacter massiliensis (43.7\% in T+ group, $p$ $<0.0000001$ ), IHU PS 91 Ruminococcaceae 1229 (33.8\% in T+ group, $p<0.0000001)$, IHU PS 90 Ruminococcaceae 24491 (30.5\% in T+ group, $p<0.0000001$ ), Ruminococcus champanellensis (31.8\% in $\mathrm{T}+$ group, $p<0.0000001)$. OTUs assigned to the Lachnospiraceae family were the following: Dorea massiliensis (40.4\% in T+ group, $p<0.0000001)$ and Roseburia intestinalis (30.5\% in T+ group, $p$ $<0.0000001)$. OTUs belonging to the Eubacteriaceae family consisted of: 
IHU PS 92 Eubacteriaceae 86603 (50.3\% in T+ group, $p<0.0000001)$, Eubacterium eligens $(64.2 \%$ in $\mathrm{T}+$ group, $p<0.0000001)$, IHU PS 92 Eubacteriaceae 18176 (50.3\% in T+ group, $p<0.0000001)$ (Figure 6).

When analyzing the metagenomes within sample types, fecal samples exhibited the same profile than the combined samples. On the other hand, oral samples presented a different profile with a lower proportion of Clostridiales (23.3\% in oral samples) in favor of Bacteroidales ( $20 \%$ vs $10 \%$ in all samples) (Figure $4 \mathrm{~A}$ ). Interestingly, members of the red complex of periodontitis were found among the 30 most frequent taxa in T+ samples namely Treponema denticola, and Tannerella forsythia (Figure 4C). The analysis restricted to rural metagenomes highlighted Treponema succinifaciens $(52 \%, p<0.0000001)$ as the second most frequent taxa in the $T+$ group and Treponema berlinense as the $6^{\text {th }}$ most frequent taxa $(50 \%, p$ $<0.0000001)$. Interestingly, two OTUs among the most frequent taxa in the $T+$ group were assigned to methanogenic archeae: Methanobrevibacter smithii (47\% in T+ group, $p<0.0000001)$ and Methanosphaera stadtmanae ( $40 \%$ in $T+$ group, $p<0.0000001$ ) (Figure $5 \mathrm{~A})$. It is noteworthy that the 30 most frequent taxa associated to the prevalence of Treponema were highly discriminant due to their low prevalence if any in the absence of Treponema spp. associated to a very low $p$ value $(p<0.0000001)$.

\section{In vitro antagonistic activity between Bifidobacterium and Bacteroides and Treponema}

The selected candidates belonged to the genera Bifidobacterium and Bacteroides as these were among the most frequent genera to emerge from our in-silico results. Therefore, Bifidobacterium bifidum CSUR P1194, Bifidobacterium longum CSUR P7400, Bifidobacterium breve CSUR P7882, Bacteroides ovatus CSUR P4577, Bacteroides uniformis CSUR P2248 and Bacteroides thetaiotaomicron CSUR P7324 were tested against Treponema denticola CSUR P7640. Inhibition control was performed using vancomycin (30 $\mu \mathrm{g}$ disc) which is an inhibitor of treponemal growth [16] and fosfomycin (50 $\mu \mathrm{g}$ disc) against which Treponema spp. are resistant for which an inhibition zone with a mean diameter of $15 \mathrm{~mm}$ (SD 0.3) was obtained (Figure 7C). After 4 to 5 days of incubation, growth inhibition of $T$. denticola was observed against $B$. longum with a mean diameter of $45 \mathrm{~mm}$ (SD 0.35), B. bifidum with a mean diameter of $40 \mathrm{~mm}$ (SD 0.8) and $B$. breve with a mean diameter of $25 \mathrm{~mm}$ (SD 0.15) (Figure 7E, 6F and 6G). Growth inhibition of $T$. denticola CSURP 7640 was also observed using Bacteroides species, namely $B$. thetaiotaomicron with a mean diameter of $25 \mathrm{~mm}$ (SD 0.45), B. uniformis with a mean diameter of $12 \mathrm{~mm}$ (SD0.15), $B$. ovatus with a mean diameter of $10 \mathrm{~mm}$ (SD 0.1) (Figure $7 \mathrm{H}, 6 \mathrm{l}$ and $6 \mathrm{~J}$ ). These results confirm the in-silico predictions as an inhibition was observed with highly frequent OTUs in the T-group (Figure 2, Table 2) while no growth inhibition was observed using fosfomycin and Prevotella rectalis (CSUR P4334) (Figure 7 D). 
Table 2

Summary table with the confirmed antagonists, their frequency in each group and the inhibition diameter obtained against Treponema denticola.

\begin{tabular}{|c|c|c|c|c|c|c|}
\hline \multirow[b]{2}{*}{ Tested species } & \multicolumn{3}{|l|}{ In silico } & \multicolumn{3}{|c|}{ In vitro } \\
\hline & $\begin{array}{l}\text { Frequency } \\
\left(T_{-}\right)\end{array}$ & $\begin{array}{l}\text { Frequency } \\
(T+)\end{array}$ & $\begin{array}{l}\text { Frequency } \\
\text { difference }\end{array}$ & P-value & $\begin{array}{l}\text { Inhibition } \\
\text { diameter } \\
(\mathrm{mm})\end{array}$ & $\begin{array}{l}\text { Standard } \\
\text { deviation }\end{array}$ \\
\hline $\begin{array}{l}\text { Bifidobacterium } \\
\text { longum }\end{array}$ & $63.3 \%$ & $15,9 \%$ & $47,4 \%$ & $\begin{array}{l}p \\
<0.0000001\end{array}$ & 45 & 0,35 \\
\hline $\begin{array}{l}\text { Bifidobacterium } \\
\text { bifidum }\end{array}$ & $25.9 \%$ & $6,0 \%$ & $20,0 \%$ & $\begin{array}{l}p \\
<0.0000001\end{array}$ & 40 & 0,8 \\
\hline $\begin{array}{l}\text { Bifidobacterium } \\
\text { breve }\end{array}$ & $21.9 \%$ & $0,7 \%$ & $21,2 \%$ & $\begin{array}{l}p \\
<0.0000001\end{array}$ & 25 & 0,15 \\
\hline $\begin{array}{l}\text { Bacteroides } \\
\text { ovatus }\end{array}$ & I & / & / & I & 10 & 0,1 \\
\hline $\begin{array}{l}\text { Bacteroides } \\
\text { thetaiotaomicron }\end{array}$ & $19.9 \%$ & $10,6 \%$ & $9,3 \%$ & $p=3,67 \mathrm{E}^{-03}$ & 25 & 0,45 \\
\hline $\begin{array}{l}\text { Bacteroides } \\
\text { uniformis }\end{array}$ & / & / & / & / & 12 & 0,15 \\
\hline
\end{tabular}

\section{Discussion}

The evolution of human lifestyle along the eras is intricately linked to that of human microbial ecosystems as the composition of the digestive tract microbiome is highly modulated by diet $[3,17]$. The changes in diet from hunter-gatherers to primitive farming in the Neolithic era and then the shift to processed food with the industrial revolution have been accompanied with many changes among which the decline of oral health $[18,19]$. In fact, the occurrence of dental caries and periodontal diseases has increased overtime and has been historically linked to unfavorable changes in western diet with the industrialization and increased consumption of processed food with high carbohydrate content [19]. Moreover, the change in feeding habits was concomitant to a decrease of the global diversity of the oral microbiota $[20,21]$ and to an increase in cariogenic species such as Streptococcus mutans, Porphyromonas gingivalis, Tannerella forsythia and Treponema spp [22]. A study by Adler et al highlighted discriminating taxa between hunter-gatherers and primitive farmers (Clostridiales and more specifically Ruminococcaceae) and between the later and modern humans (Veillonellaceae, Lachnospiraceae, Actinomycetales). These findings are consistent with our results that highlight a high proportion of Veillonellaceae and Actinomycetales among the most frequent taxa in the absence of Treponema spp. in oral samples.

Our study shows in fecal samples an anticorrelation of the prevalence in Treponema spp. with that of Bifidobacterium spp., Streptococcus spp. and Bacteroides spp. which were the most represented among the most frequent taxa in metagenomes not prevalent in treponemes. This anticorrelation was confirmed 
in vitro through the growth inhibition of $T$. denticola CSUR P7640. For the first time, strains belonging to the Bifidobacterium and Bacteroides genera showed an activity against $T$. denticola. Within the same genus, the antimicrobial activity against $T$. denticola was species dependent and positively correlated with the frequency of the species in T- metagenomes with $B$. longum CSUR P7400 exhibiting the highest anti-treponemal activity.

Bifidobacteria are part of the extensively studied group of lactic acid bacteria [23] which use have evolved with human lifestyle. Lactic acid bacteria were used early on for fermentation and preservation of foodstuffs [24-26]. Even though the term of lactic acid bacteria dates to the 1900s, very early reports (as early as $3200 \mathrm{BC}$ ) state that fermented food have been a part of human diet [23, 27]. With the increased need for food storage and conservation, the modern era has seen an increased use of lactic acid bacteria as bio-preservatives with the transition from hunting and gathering to the agricultural lifestyle and finally the industrial era [27]. In the 1900s, Metchnikoff introduced the world to the use of "friendly" bacteria [28] presently known as probiotics [29] to improve health. Using dairy products, probiotics are nowadays widely used by western populations through the consumption of fermented food, biopreserved food as well as intended consumption of lactic acid bacteria thus providing numerous health benefits [30, 31]. Similarly, to the diet-induced changes of the oral microbiome, this increased consumption of lactic acid bacteria seems to coincide with the loss of treponemes in urban populations with a diet dominated by processed food. In fact, populations which consume processed food-based diet retain bifidobacteria conversely to populations with a diet consisting of local products or prehistoric populations $[2,5,15]$. This observation could provide a possible mechanism underlying the loss of treponemes in urban populations. As suggested by several studies, the total absence of treponemes in western populations could indeed be linked to the evolution of lifestyle with the dietary changes associated to the phenomenon of nutrition transition and increased use of probiotics due to the shift in the medical concepts of bacteria from exclusive foe to potential friend.

\section{Conclusions}

Our study clearly highlights Bifidobacterium spp. and Bacteroides spp. as antagonists of Treponema spp. through an in silico analysis which predictions were confirmed in vitro. This antagonism, reported here for the first time, provides a novel hypothesis regarding the loss of treponemes in the gut microbiota of western populations which may be linked to the increased prevalence in bifidobacteria associated with the consumption of processed foods and the use of probiotics.

\section{Declarations}

\section{Acknowlegdements}

We wish to thank the Sequencing and Bioinformatics plateforms of the Méditerrannée Infection University Hospital for processing the analyzed metagenomes. 


\section{Funding}

This work has received financial support from the Méditerranée Infection and the French Government through the Agence Nationale pour la Recherche (ANR), including the "Programme d'Investissement d'Avenir" under the reference Méditerranée Infection 10-IAHU-03.

\section{Authors'contributions}

Conceptualization: DR. Funding acquisition: DR. Data curation and analysis: SB, MTA, MM, AL and SK. Visualization: SB and MTA. Manuscript writing: SB, MTA and SK. Supervision: SK, MTA and DR. Manuscript reviewing and editing: all.

\section{Availability of data and material}

The raw data of this study are provided in the supplementary file.

\section{Ethics approval and consent to participate}

Written consent was obtained for each participant of this study and the study obtained the ethical approval of the relevant institution as stated in the "samples" section of the methods.

\section{Consent for publication}

Not applicable.

\section{Competing interests}

No competing interests to declare.

\section{References}

1. Gomez A, Petrzelkova K, Yeoman CJ, Burns MB, Amato KR, Vlckova K, et al. Ecological and evolutionary adaptations shape the gut microbiome of BaAka African rainforest hunter-gatherers. bioRxiv [Internet]. 2015 [cited 2019 Nov 25]; Available from: http://biorxiv.org/lookup/doi/10.1101/019232

2. Segata N. Gut Microbiome: Westernization and the Disappearance of Intestinal Diversity. Current Biology. 2015;25:R611-3. 
3. De Filippo C, Cavalieri D, Di Paola M, Ramazzotti M, Poullet JB, Massart S, et al. Impact of diet in shaping gut microbiota revealed by a comparative study in children from Europe and rural Africa. Proc Natl Acad Sci U S A. 2010;107:14691-6.

4. Rampelli S, Schnorr SL, Consolandi C, Turroni S, Severgnini M, Peano C, et al. Metagenome Sequencing of the Hadza Hunter-Gatherer Gut Microbiota. Current Biology. 2015;25:1682-93.

5. Schnorr SL, Candela M, Rampelli S, Centanni M, Consolandi C, Basaglia G, et al. Gut microbiome of the Hadza hunter-gatherers. Nature Communications [Internet]. 2014 [cited 2017 Oct 22];5. Available from: http://www.nature.com/doifinder/10.1038/ncomms4654

6. Angelakis E, Bachar D, Yasir M, Musso D, Djossou F, Gaborit B, et al. Treponema species enrich the gut microbiota of traditional rural populations but are absent from urban individuals. New Microbes and New Infections. 2019;27:14-21.

7. Tidjani Alou M, Lagier J-C, Raoult D. Diet influence on the gut microbiota and dysbiosis related to nutritional disorders. Human Microbiome Journal. 2016;1:3-11.

8. Belkacemi S, Boukhalil J, Ominami Y, Hisada A, Fontanini A, Caputo A, et al. Passive filtration, rapid scanning electron microscopy and MALDI-TOF MS for Treponema culture and identification from the oral cavity. Journal of Clinical Microbiology [Internet]. 2019 [cited 2019 Sep 4]; Available from: http://jcm.asm.org/lookup/doi/10.1128/JCM.00517-19

9. Angelakis E, Bachar D, Yasir M, Musso D, Djossou F, Melenotte C, et al. Comparison of the gut microbiota of obese individuals from different geographic origins. New microbes and new infections. 2019;27:40-7.

10. Morton ER, Lynch J, Froment A, Lafosse S, Heyer E, Przeworski M, et al. Variation in Rural African Gut Microbiota Is Strongly Correlated with Colonization by Entamoeba and Subsistence. PLoS Genet [Internet]. 2015 [cited 2018 Dec 3];11. Available from: https://www.ncbi.nlm.nih.gov/pmc/articles/PMC4664238/

11. Yatsunenko T, Rey FE, Manary MJ, Trehan I, Dominguez-Bello MG, Contreras M, et al. Human gut microbiome viewed across age and geography. Nature. 2012;486:222-7.

12. Soverini M, Rampelli S, Turroni S, Schnorr SL, Quercia S, Castagnetti A, et al. Variations in the Postweaning Human Gut Metagenome Profile As Result of Bifidobacterium Acquisition in the Western Microbiome. Front Microbiol. 2016;7:1058.

13. Angelakis E, Yasir M, Bachar D, Azhar El, Lagier J-C, Bibi F, et al. Gut microbiome and dietary patterns in different Saudi populations and monkeys. Scientific Reports. 2016;6:32191.

14. Obregon-Tito AJ, Tito RY, Metcalf J, Sankaranarayanan K, Clemente JC, Ursell LK, et al. Subsistence strategies in traditional societies distinguish gut microbiomes. Nature Communications. 2015;6:6505.

15. Tito RY, Knights D, Metcalf J, Obregon-Tito AJ, Cleeland L, Najar F, et al. Insights from Characterizing Extinct Human Gut Microbiomes. PLoS One [Internet]. 2012 [cited 2018 Dec 3];7. Available from: https://www.ncbi.nlm.nih.gov/pmc/articles/PMC3521025/ 
16. Abramson IJ, Smibert RM. Inhibition of growth of treponemes by antimicrobial agents. Sexually Transmitted Infections. 1971;47:407-12.

17. Barone M, Turroni S, Rampelli S, Soverini M, D'Amico F, Biagi E, et al. Gut microbiome response to a modern Paleolithic diet in a Western lifestyle context. Loor JJ, editor. PLoS ONE. 2019;14:e0220619.

18. Sajantila A. Major historical dietary changes are reflected in the dental microbiome of ancient skeletons. Invest Genet. 2013;4:10.

19. Adler CJ, Dobney K, Weyrich LS, Kaidonis J, Walker AW, Haak W, et al. Sequencing ancient calcified dental plaque shows changes in oral microbiota with dietary shifts of the Neolithic and Industrial revolutions. Nat Genet. 2013;45:450-5.

20. Raoult D, Foti B, Aboudharam G. Historical and geographical parallelism between the incidence of dental caries, Streptococcus mutans and sugar intake. Eur J Epidemiol. 2013;28:709-10.

21. Parker EJ, Jamieson LM, Broughton J, Albino J, Lawrence HP, Roberts-Thomson K. The oral health of Indigenous children: A review of four nations: Oral health of Indigenous children. Journal of Paediatrics and Child Health. 2010;46:483-6.

22. Mohanty R, Asopa SJ, Joseph MD, Singh B, Rajguru JP, Saidath K, et al. Red complex: Polymicrobial conglomerate in oral flora: A review. J Family Med Prim Care. 2019;8:3480-6.

23. Stiles ME, Holzapfel WH. Lactic acid bacteria of foods and their current taxonomy. International Journal of Food Microbiology. 1997;36:1-29.

24. Leroy F, De Vuyst L. Lactic acid bacteria as functional starter cultures for the food fermentation industry. Trends in Food Science \& Technology. 2004;15:67-78.

25. Crowley S, Mahony J, van Sinderen D. Current perspectives on antifungal lactic acid bacteria as natural bio-preservatives. Trends in Food Science \& Technology. 2013;33:93-109.

26. Schnürer J, Magnusson J. Antifungal lactic acid bacteria as biopreservatives. Trends in Food Science \& Technology. 2005;16:70-8.

27. Douillard FP, de Vos WM. Functional genomics of lactic acid bacteria: from food to health. Microb Cell Fact. 2014;13:S8.

28. Podolsky SH. Metchnikoff and the microbiome. The Lancet. 2012;380:1810-1.

29. Fuller R. History and development of probiotics. Probiotics [Internet]. Dordrecht: Springer Netherlands; 1992 [cited 2020 Sep 9]. p. 1-8. Available from: http://link.springer.com/10.1007/978-94-011-23648_1

30. Pasolli E, De Filippis F, Mauriello IE, Cumbo F, Walsh AM, Leech J, et al. Large-scale genome-wide analysis links lactic acid bacteria from food with the gut microbiome. Nat Commun. 2020;11:2610.

31. Markowiak P, Śliżewska K. Effects of Probiotics, Prebiotics, and Synbiotics on Human Health. Nutrients [Internet]. 2017 [cited 2020 Sep 10];9. Available from: https://www.ncbi.nlm.nih.gov/pmc/articles/PMC5622781/

32. Masella AP, Bartram AK, Truszkowski JM, Brown DG, Neufeld JD. PANDAseq: paired-end assembler for illumina sequences. BMC Bioinformatics. 2012;13:31. 
33. Edgar RC, Haas BJ, Clemente JC, Quince C, Knight R. UCHIME improves sensitivity and speed of chimera detection. Bioinformatics. 2011;27:2194-200.

34. Edgar RC. Search and clustering orders of magnitude faster than BLAST. Bioinformatics. 2010;26:2460-1.

35. Caporaso JG, Kuczynski J, Stombaugh J, Bittinger K, Bushman FD, Costello EK, et al. QIIME allows analysis of high-throughput community sequencing data. Nature Methods. 2010;7:335-6.

36. Stoeck T, Behnke A, Christen R, Amaral-Zettler L, Rodriguez-Mora MJ, Chistoserdov A, et al. Massively parallel tag sequencing reveals the complexity of anaerobic marine protistan communities. BMC Biology. 2009;7:72.

37. Mondani L, Piette L, Christen R, Bachar D, Berthomieu C, Chapon V. Microbacterium lemovicicum sp. nov., a bacterium isolated from a natural uranium-rich soil. International Journal of Systematic and Evolutionary Microbiology. 2013;63:2600-6.

38. Boissière A, Tchioffo MT, Bachar D, Abate L, Marie A, Nsango SE, et al. Midgut Microbiota of the Malaria Mosquito Vector Anopheles gambiae and Interactions with Plasmodium falciparum Infection. Vernick KD, editor. PLoS Pathogens. 2012;8:e1002742.

39. Altschul SF, Gish W, Miller W, Myers EW, Lipman DJ. Basic local alignment search tool. Journal of Molecular Biology. 1990;215:403-10.

40. Yilmaz P, Parfrey LW, Yarza P, Gerken J, Pruesse E, Quast C, et al. The SILVA and "All-species Living Tree Project (LTP)" taxonomic frameworks. Nucleic Acids Research. 2014;42:D643-8.

41. Quast C, Pruesse E, Yilmaz P, Gerken J, Schweer T, Yarza P, et al. The SILVA ribosomal RNA gene database project: improved data processing and web-based tools. Nucleic Acids Research. 2012;41:D590-6.

42. Weiss S, Xu ZZ, Peddada S, Amir A, Bittinger K, Gonzalez A, et al. Normalization and microbial differential abundance strategies depend upon data characteristics. Microbiome [Internet]. 2017 [cited 2020 Feb 10];5. Available from: http://microbiomejournal.biomedcentral.com/articles/10.1186/s40168-017-0237-y

43. Waksman SA. Microbial Antagonisms and Antibiotic Substances. Microbial Antagonisms and Antibiotic Substances [Internet]. 1947 [cited 2019 Dec 19]; Available from: https://www.cabdirect.org/cabdirect/abstract/19482702475

\section{Figures}




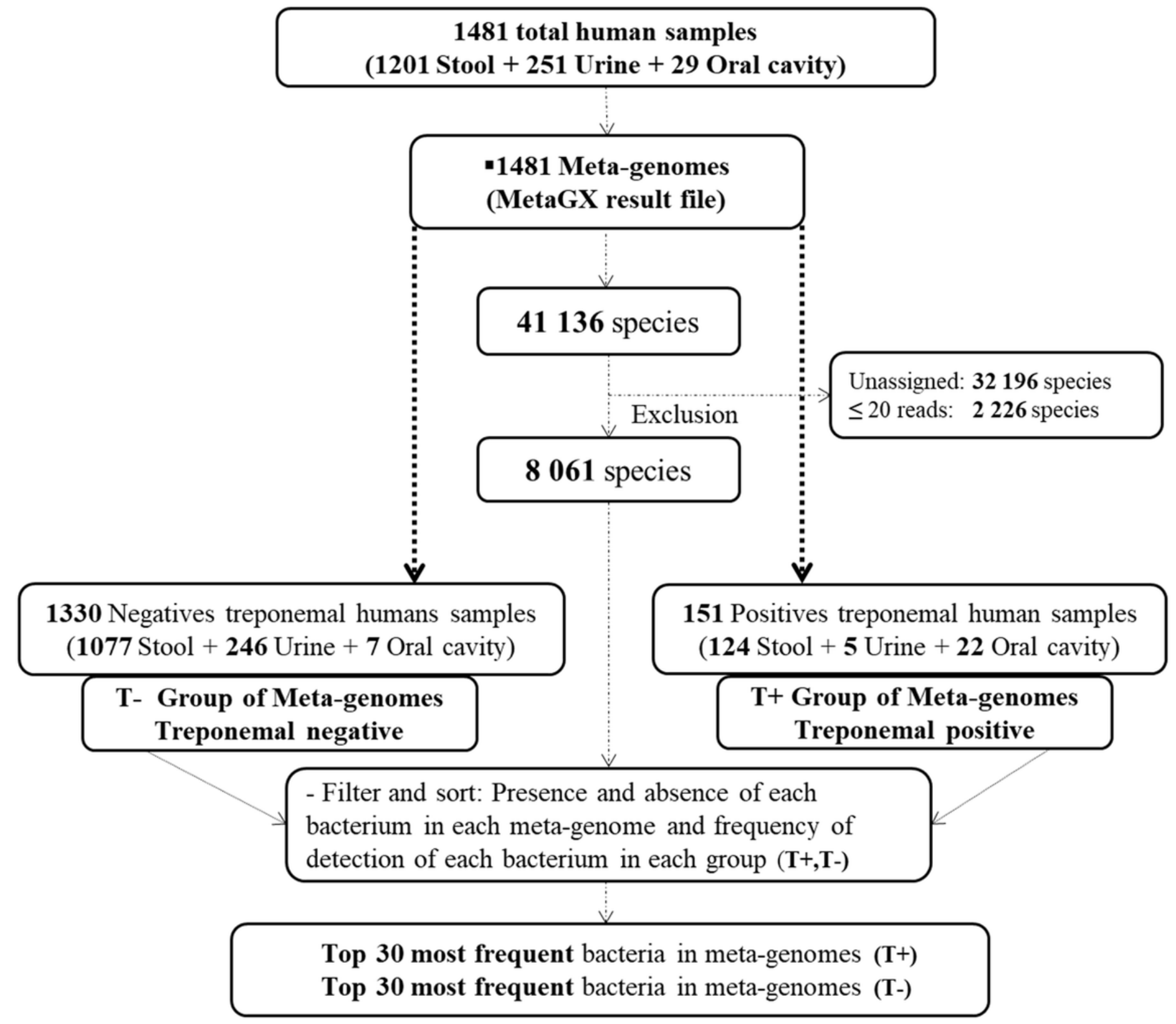

Figure 1

In-silico methodology applied in this study. 


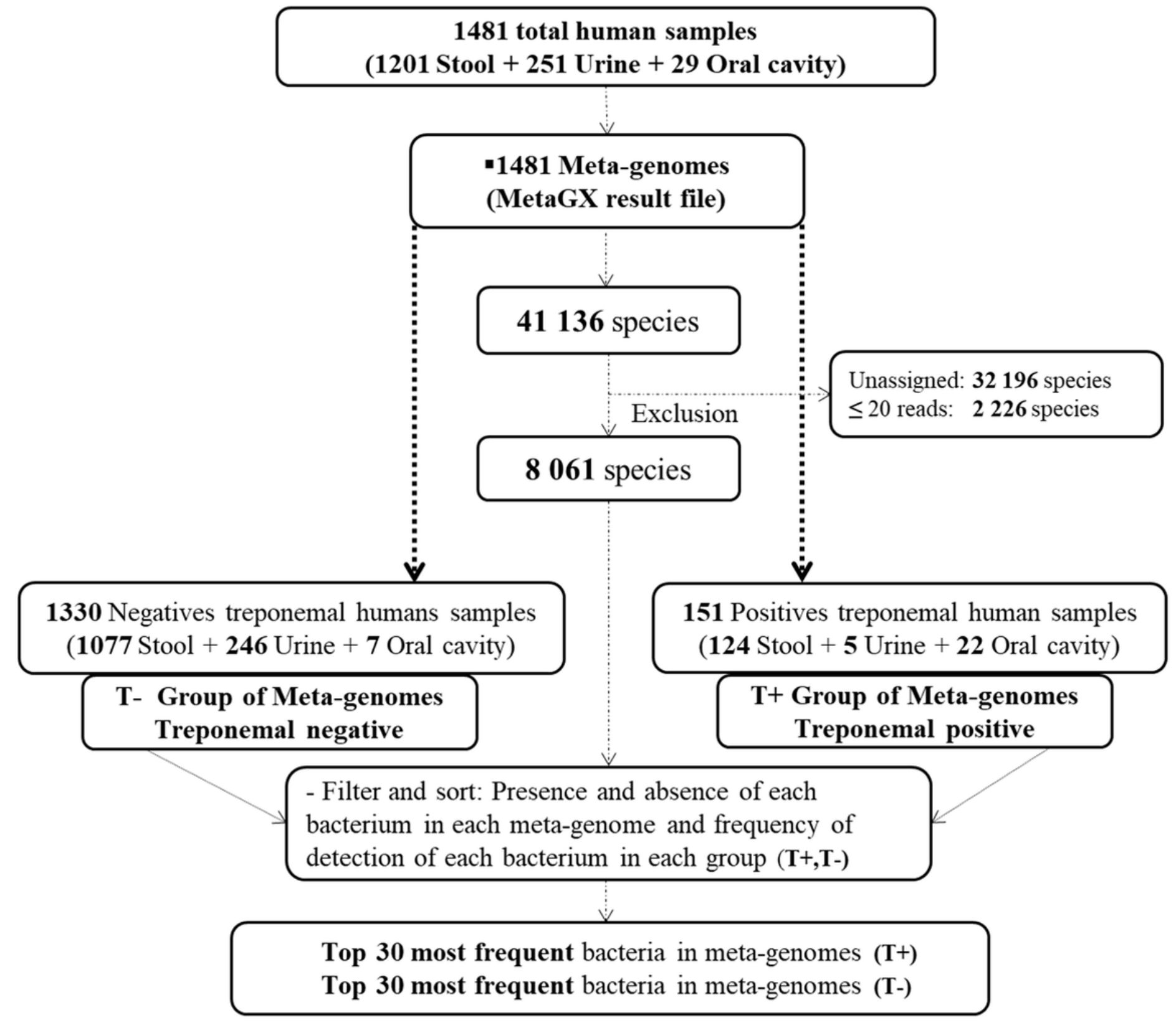

Figure 1

In-silico methodology applied in this study. 


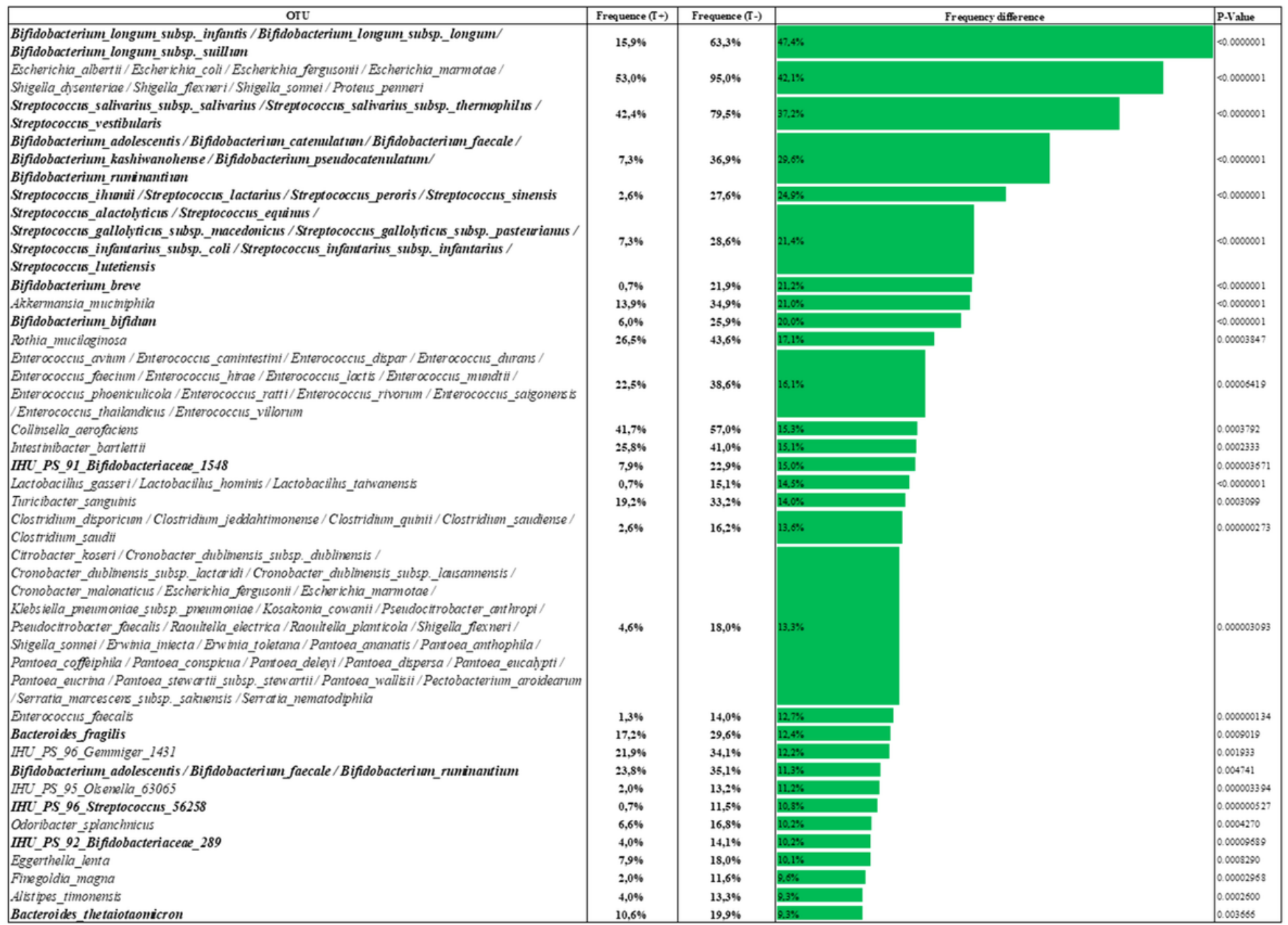

\section{Figure 2}

Top ranking predicted antagonists. The frequency difference of each OTU was calculated between the metagenomes in which Treponema spp. were not detected (T- group) and the metagenomes prevalent in Treponema spp. ( $T+$ group). The 30 most frequent OTUs in the T- group are represented in this figure with their respective frequency in the $T+$ and $T$ - metagenomes as well as the associated $p$ value. 


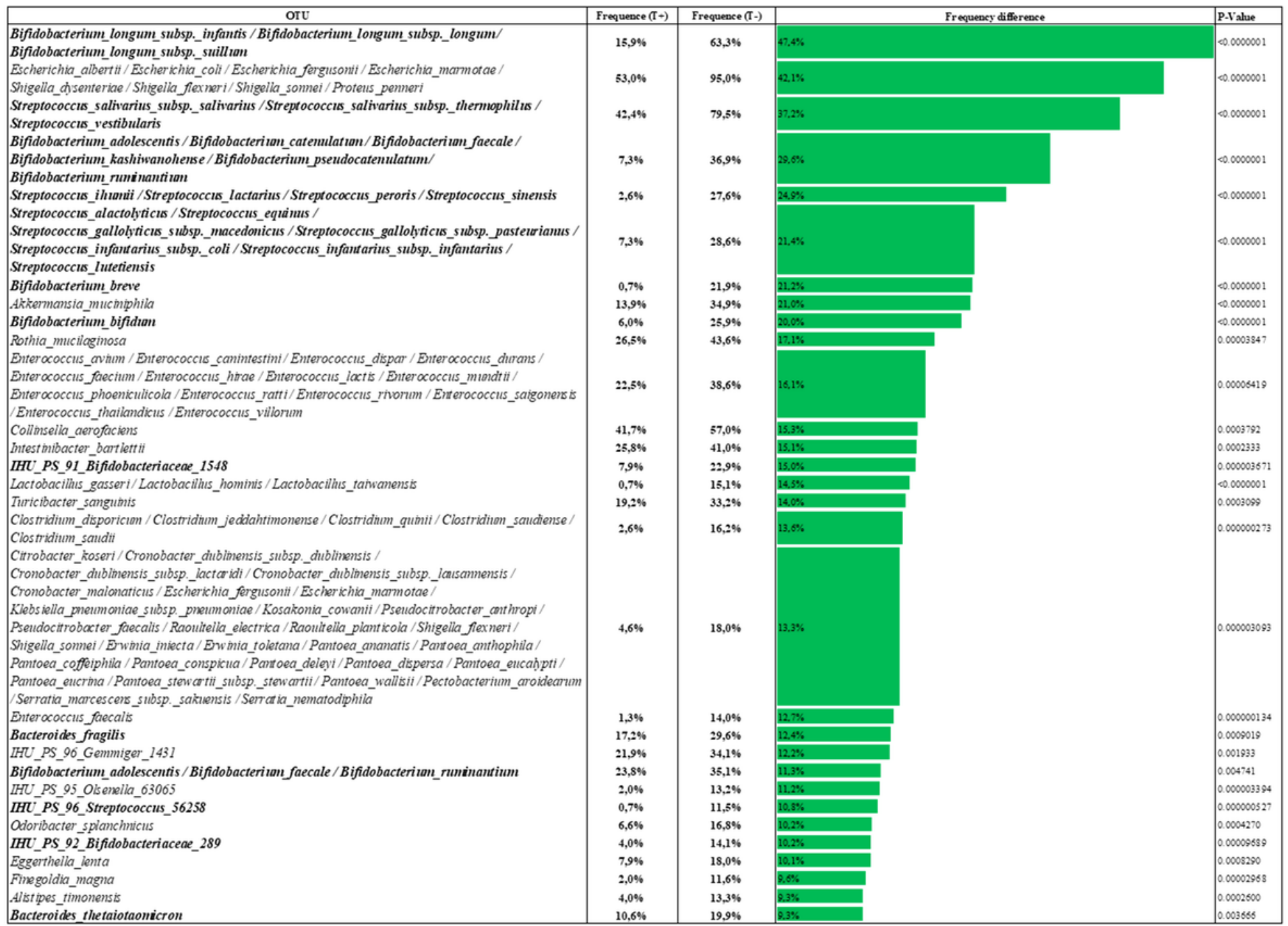

\section{Figure 2}

Top ranking predicted antagonists. The frequency difference of each OTU was calculated between the metagenomes in which Treponema spp. were not detected (T- group) and the metagenomes prevalent in Treponema spp. ( $T+$ group). The 30 most frequent OTUs in the T- group are represented in this figure with their respective frequency in the $T+$ and $T$ - metagenomes as well as the associated $p$ value.

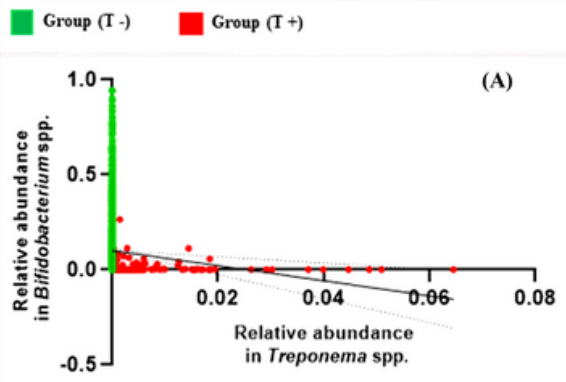

All Samples

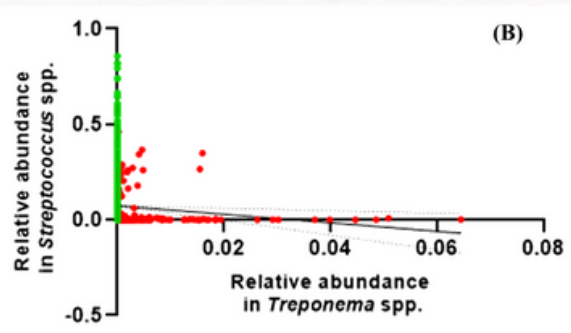

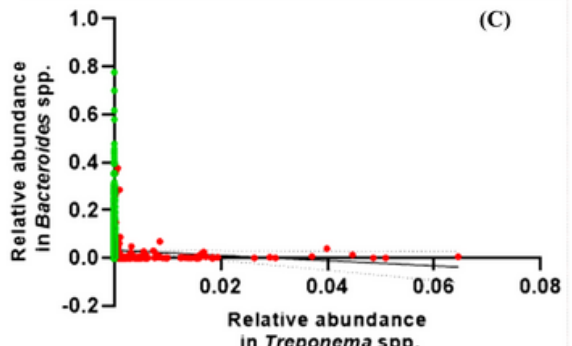


Anti-correlation between Treponema spp and Bifidobacterium spp., Streptococcus spp. and Bacteroides spp. The relative abundance of Treponema spp. in all the metagenomes included in this study was anticorrelated with that of (A) Bifidobacterium spp. $(r 2=-0,2016, p<0.0001)$, (B) Streptococcus spp. $(r 2=-0,2557, p<0.0001)$ and $(C)$ Bacteroides spp. $(r 2=-0,04911, p=0.05)$.
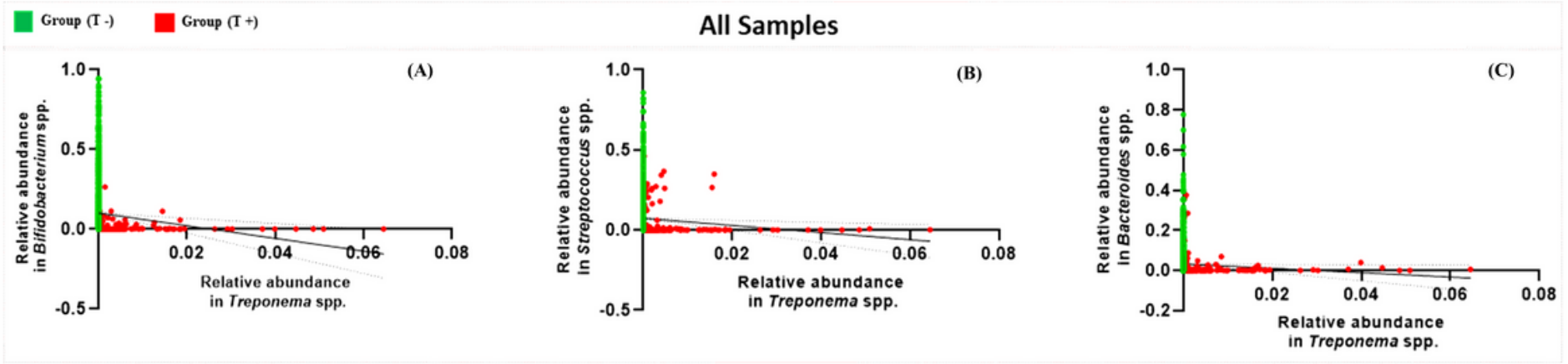

\section{Figure 3}

Anti-correlation between Treponema spp and Bifidobacterium spp., Streptococcus spp. and Bacteroides spp. The relative abundance of Treponema spp. in all the metagenomes included in this study was anticorrelated with that of (A) Bifidobacterium spp. ( $r 2=-0,2016, p<0.0001)$, (B) Streptococcus spp. $(r 2=-0,2557, p<0.0001)$ and $(C)$ Bacteroides spp. $(r 2=-0,04911, p=0.05)$. 

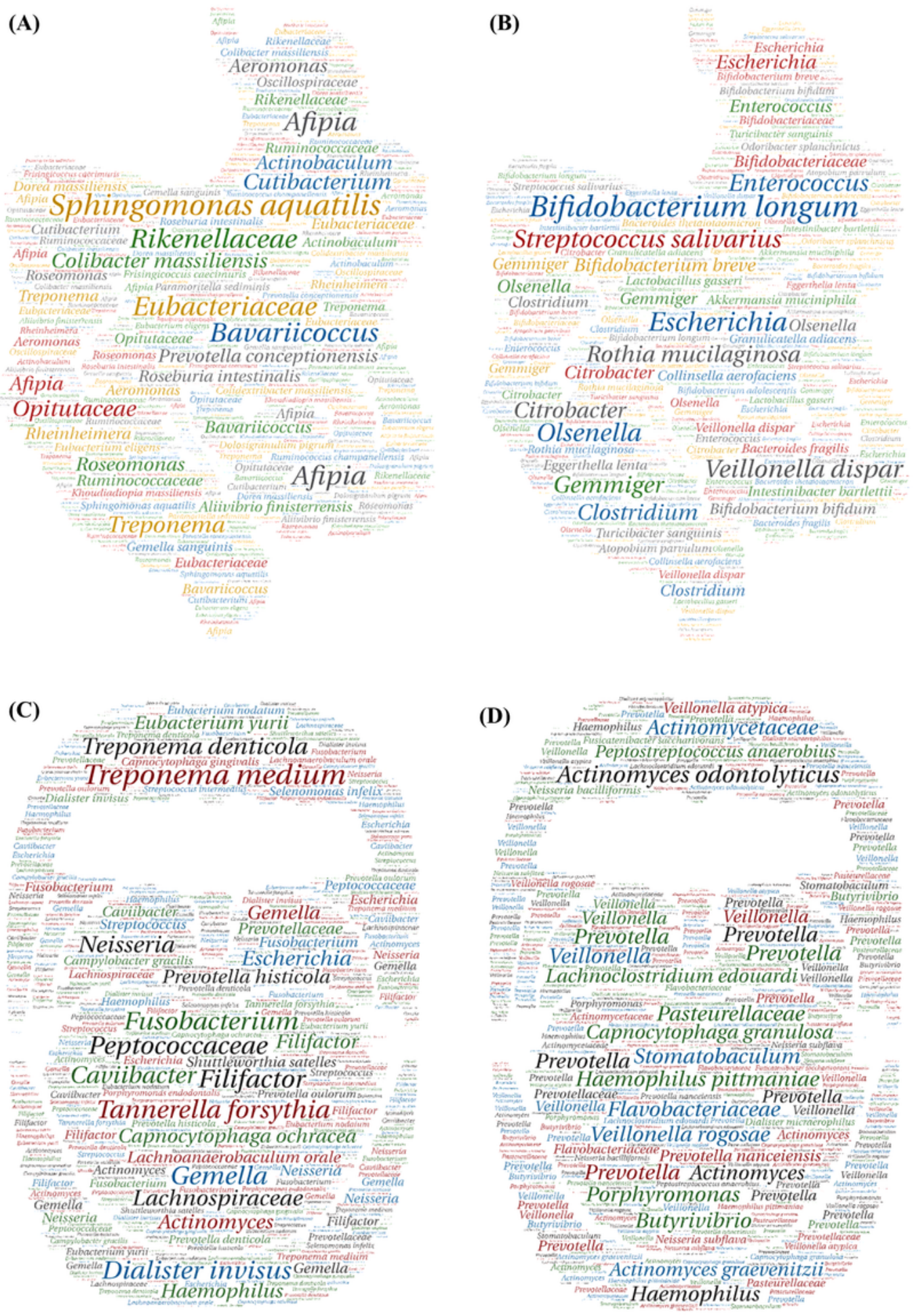

\section{Figure 4}

The frequency of bacterial species in T- group and T+ group of fecal and oral metagenomes. OTUs are represented according to their detection rate in each group using the wordart website (https://wordart.com/my-word-art). (A) Fecal metagenomes in which Treponema spp. were detected. (B) Fecal metagenomes in which Treponema spp. were not detected. (C) Oral cavity metagenomes in which Treponema spp. were detected. (D) Oral metagenomes in which Treponema spp. were not detected. 

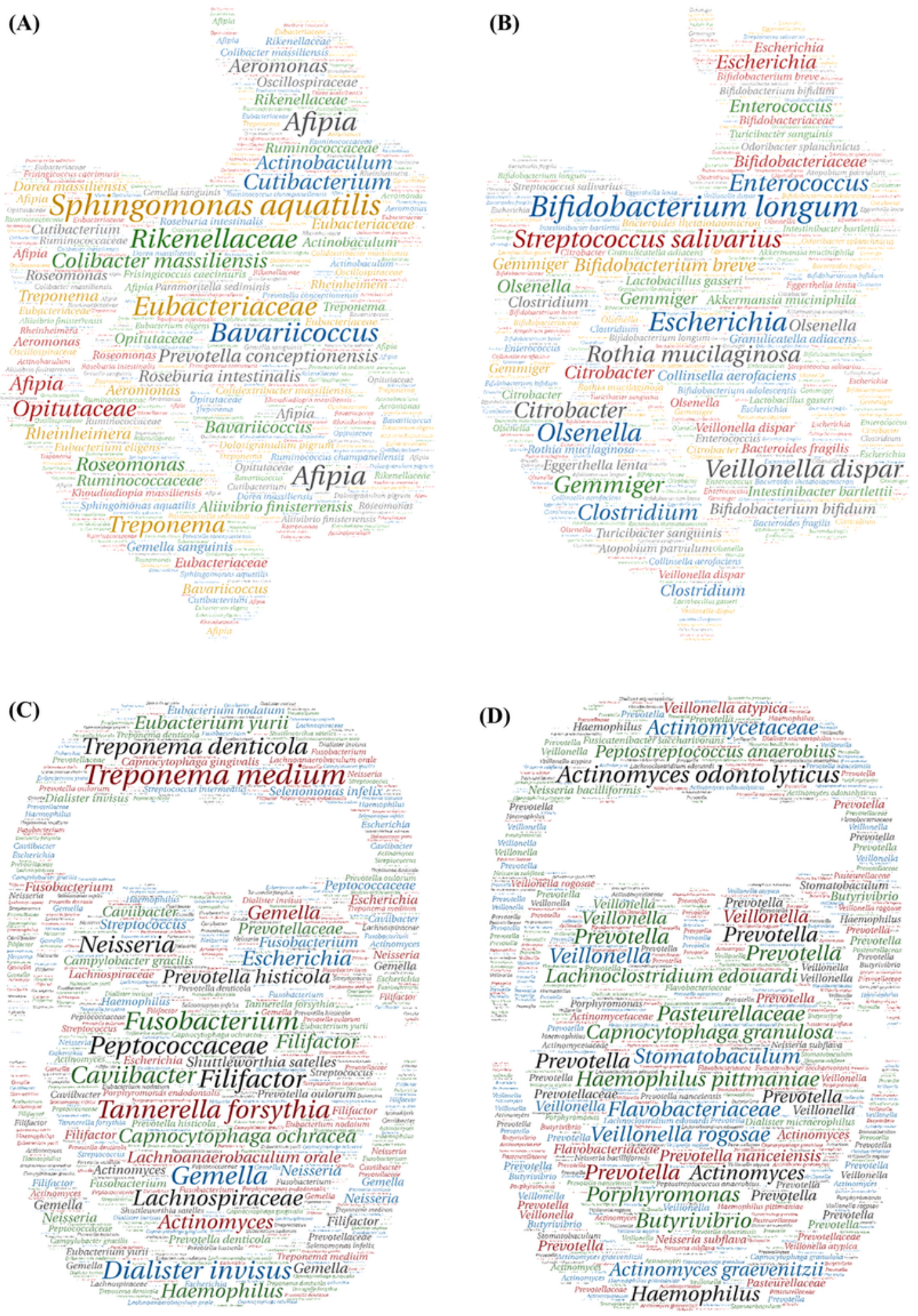

\section{Figure 4}

The frequency of bacterial species in T- group and T+ group of fecal and oral metagenomes. OTUs are represented according to their detection rate in each group using the wordart website (https://wordart.com/my-word-art). (A) Fecal metagenomes in which Treponema spp. were detected. (B) Fecal metagenomes in which Treponema spp. were not detected. (C) Oral cavity metagenomes in which Treponema spp. were detected. (D) Oral metagenomes in which Treponema spp. were not detected. 
(A)

orea massiliensis

Methanosphaera_stadtmanae IHUU PS 92 Lachnospiraceae_463"

Paramoritella_sediminis Gemella_sanguinis
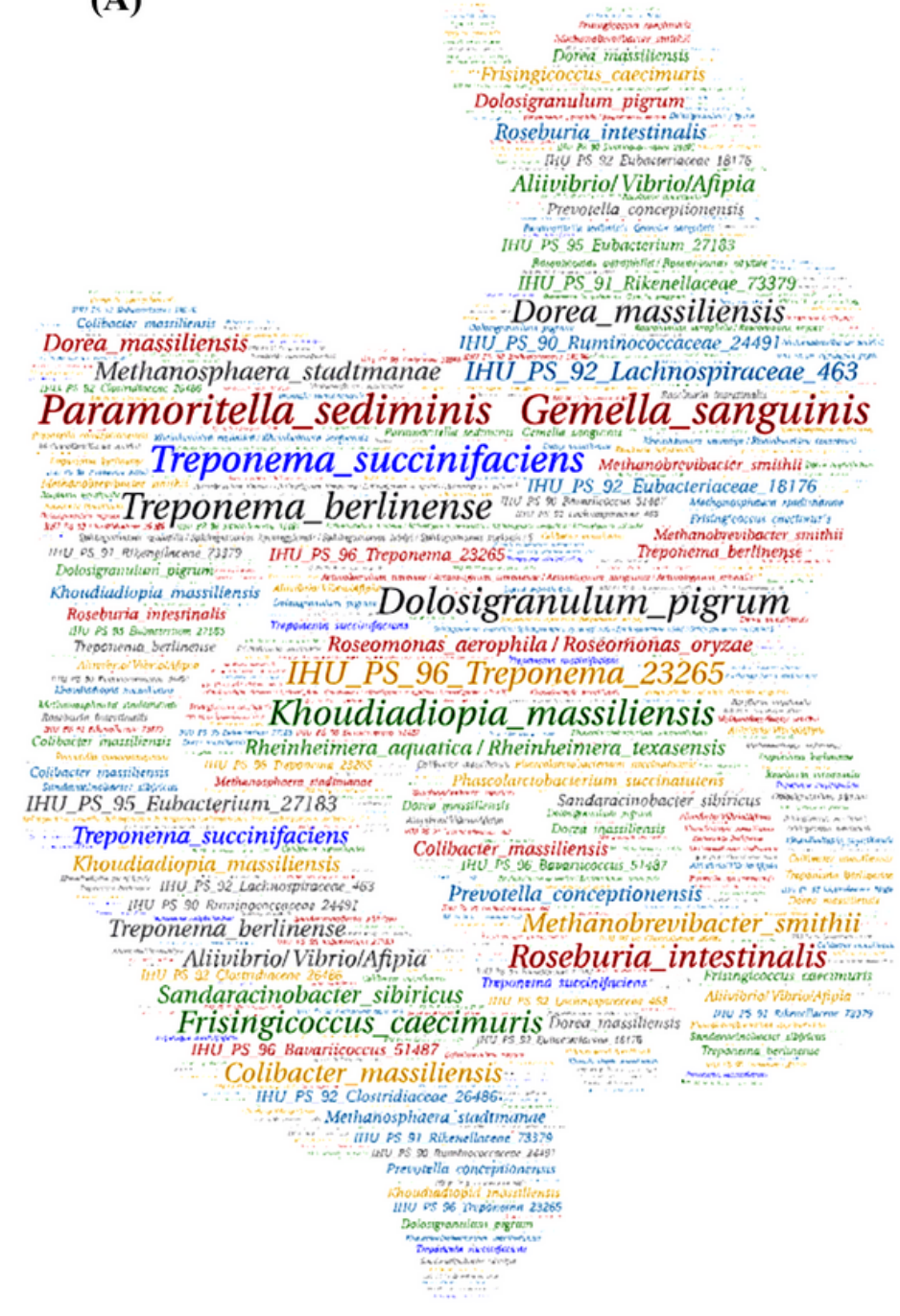

Dorea_massiliensis
IHU $P S .90$ Runinococcacene 24491

IHU PS 92 Lachnospiraceae 463
(B)

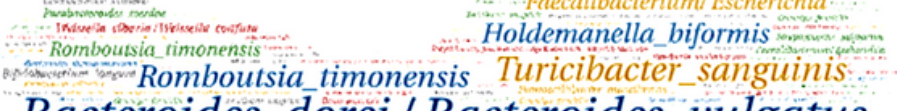

Bacteroides_dorei / Bacteroides_vulgatus

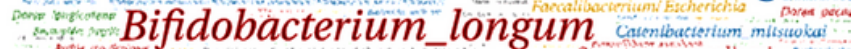

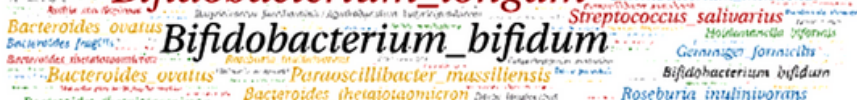

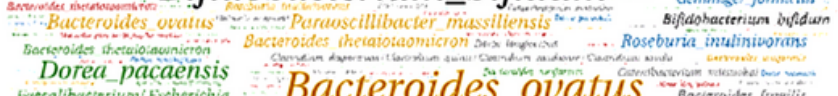

Eubacterian halli

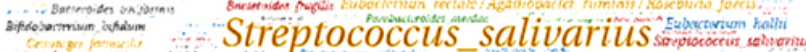

Bacteroides thetaiotaomicron

contepiger fornitilis Dorea longicatena Catenibacterium mitsuokai

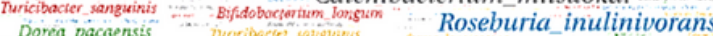

Dórea_pacaensis

Bacteroides fragilis

Rothia_mucilaginosa

Bifidobacterium bifidum

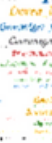

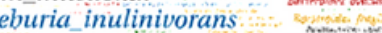

Eubacterium_hallit. Collinsella_aerofaciens Weissella cibaria / Weissella confusa Bacteroides fragilis șrepioconctur saliour iuts Parabacteroides_merdâe

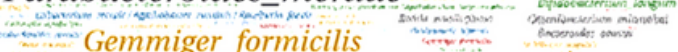

- . wew Gemiger formicilis

Collinsella aerofaciens -..... Bacteroides uniformis: Collinsella aerofaciens

Hoidemanelita biforit

Dorea longtoutena

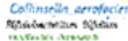

Figure 5

The frequency of bacterial species in T- group and T+ group of fecal metagenomes from individuals with a rural lifestyle. OTUs are represented according to their detection rate in each group using the wordart website (https://wordart.com/my-word-art). (A) Rural metagenomes in which Treponema spp. were detected. (B) Rural metagenomes in which Treponema spp. were not detected. 
(A)

Dorea massiliensis mathis

Methanosphaera_stadtmanae IHU_PS_92_Lachnospiraceae_463

Paramoritella_sediminis Gemella_sanguinis

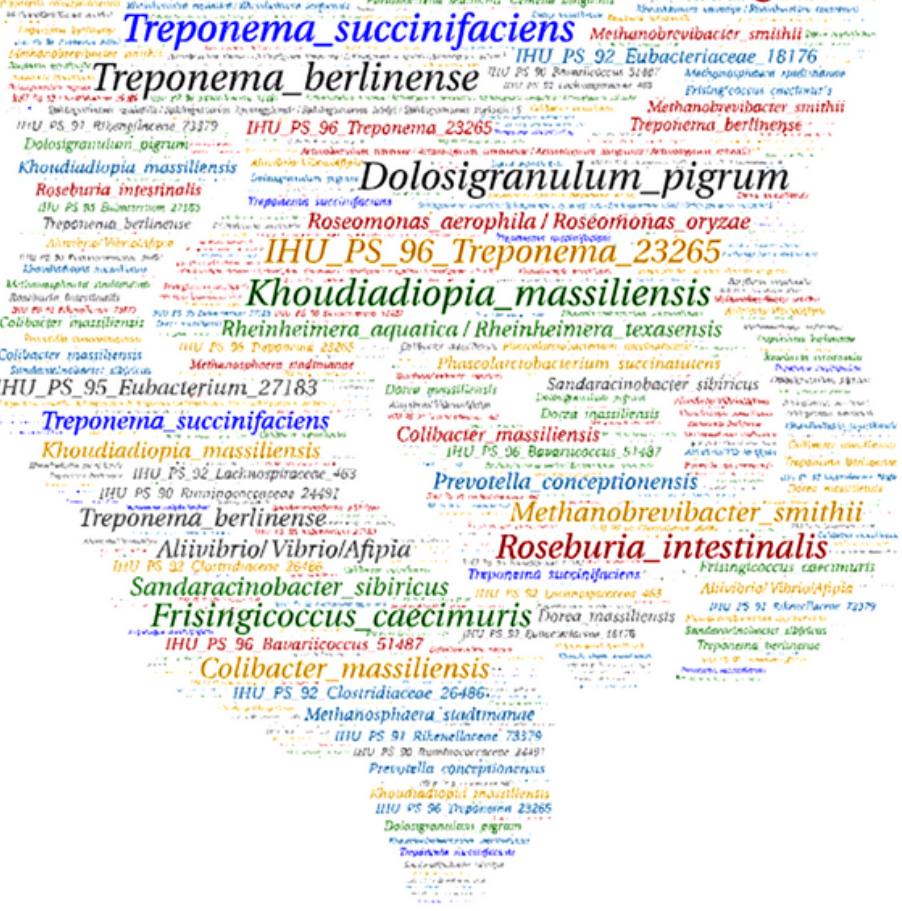

(B)

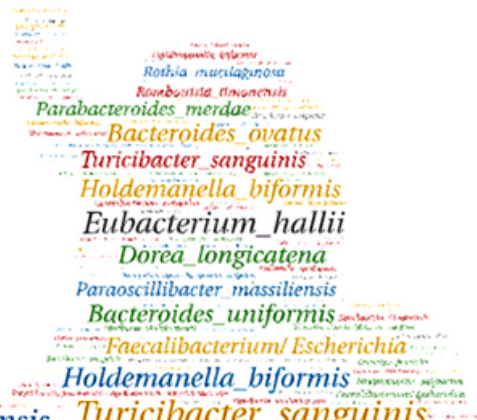

Romboutsia timonensis

Romboutsia_timonensis Turicibacter_sanguinis

Bacteroides_dorei / Bacteroides_vulgatus

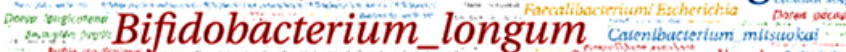

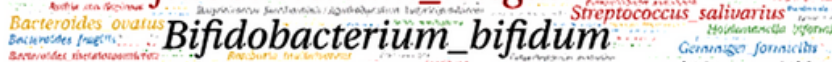

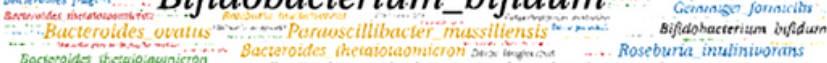

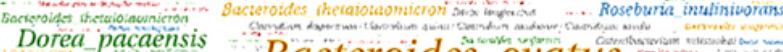

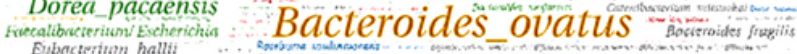

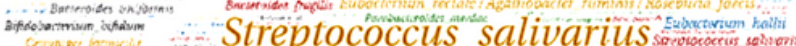

Bacteroides thetaiotaomicron

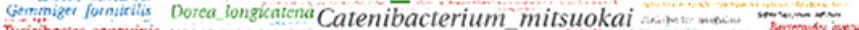

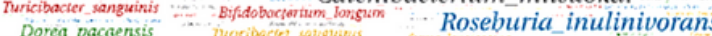

Dorea_pacaensis

Bacteroides fragilis

Rothia_mucilaginosa

Bifidobacterium bificlum

Bubacterium_hallit Collinsella_aerofaciens

$\cos _{i \rightarrow \infty}$ s.

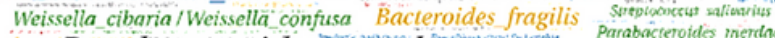
Parabacteroides_merdae Gemmiger formicilis and

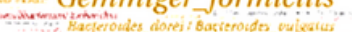

Collinsella aerafacients.-. Bacteroides uniformisi Collinsella aerofaciens

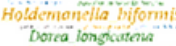

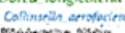

Figure 5

The frequency of bacterial species in T- group and T+ group of fecal metagenomes from individuals with a rural lifestyle. OTUs are represented according to their detection rate in each group using the wordart website (https://wordart.com/my-word-art). (A) Rural metagenomes in which Treponema spp. were detected. (B) Rural metagenomes in which Treponema spp. were not detected. 


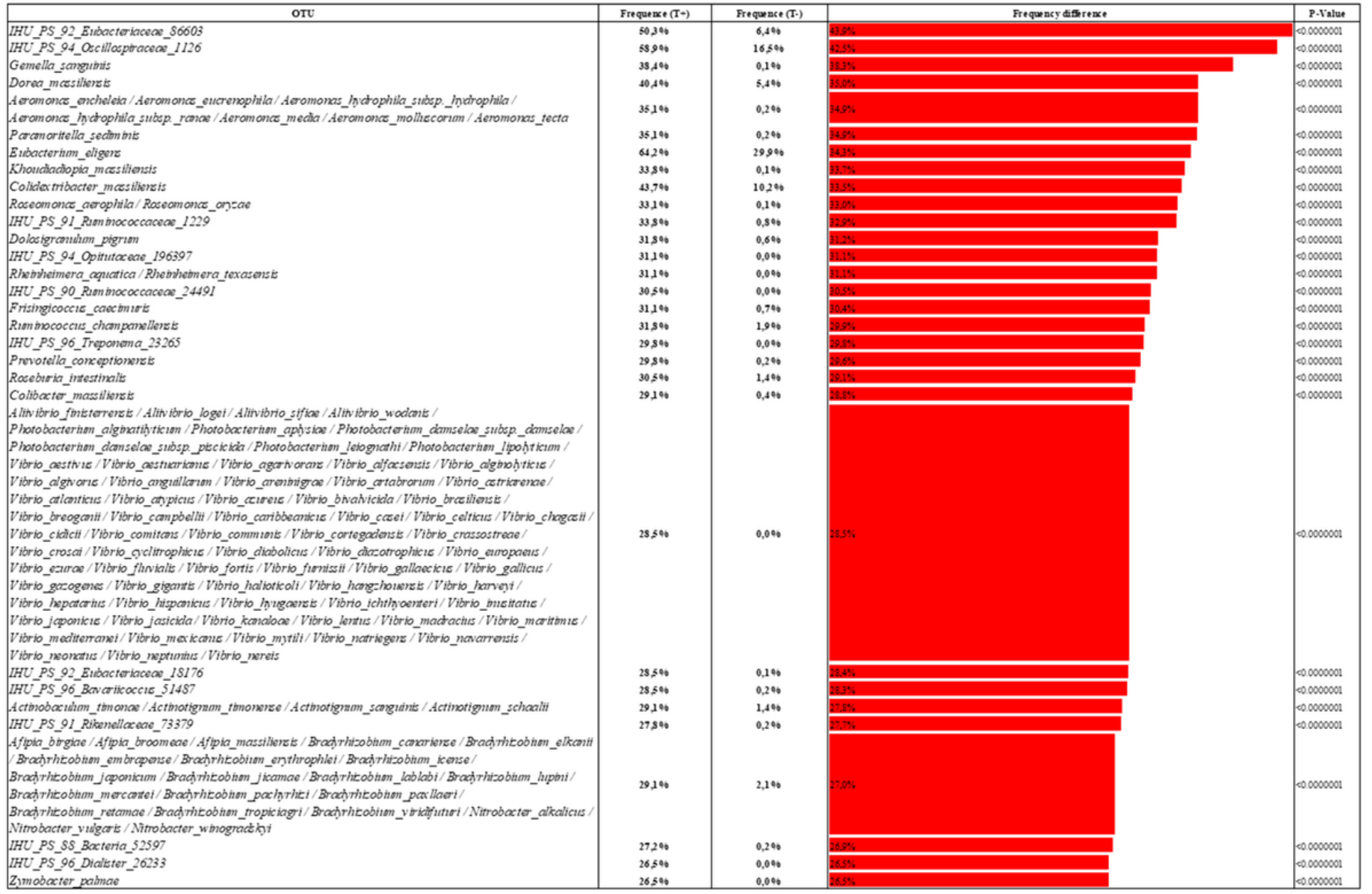

\section{Figure 6}

Most frequent OTUs associated to the prevalence in Treponema spp. The frequency difference of each OTU was calculated between the metagenomes prevalent in Treponema spp. ( $T+$ group) and the metagenomes in which Treponema spp. were not detected (T- group). The 30 most frequent OTUs in the $T+$ group are represented in this figure with their respective frequency in the $T+$ and $T$ - metagenomes as well as the associated $p$ value. 


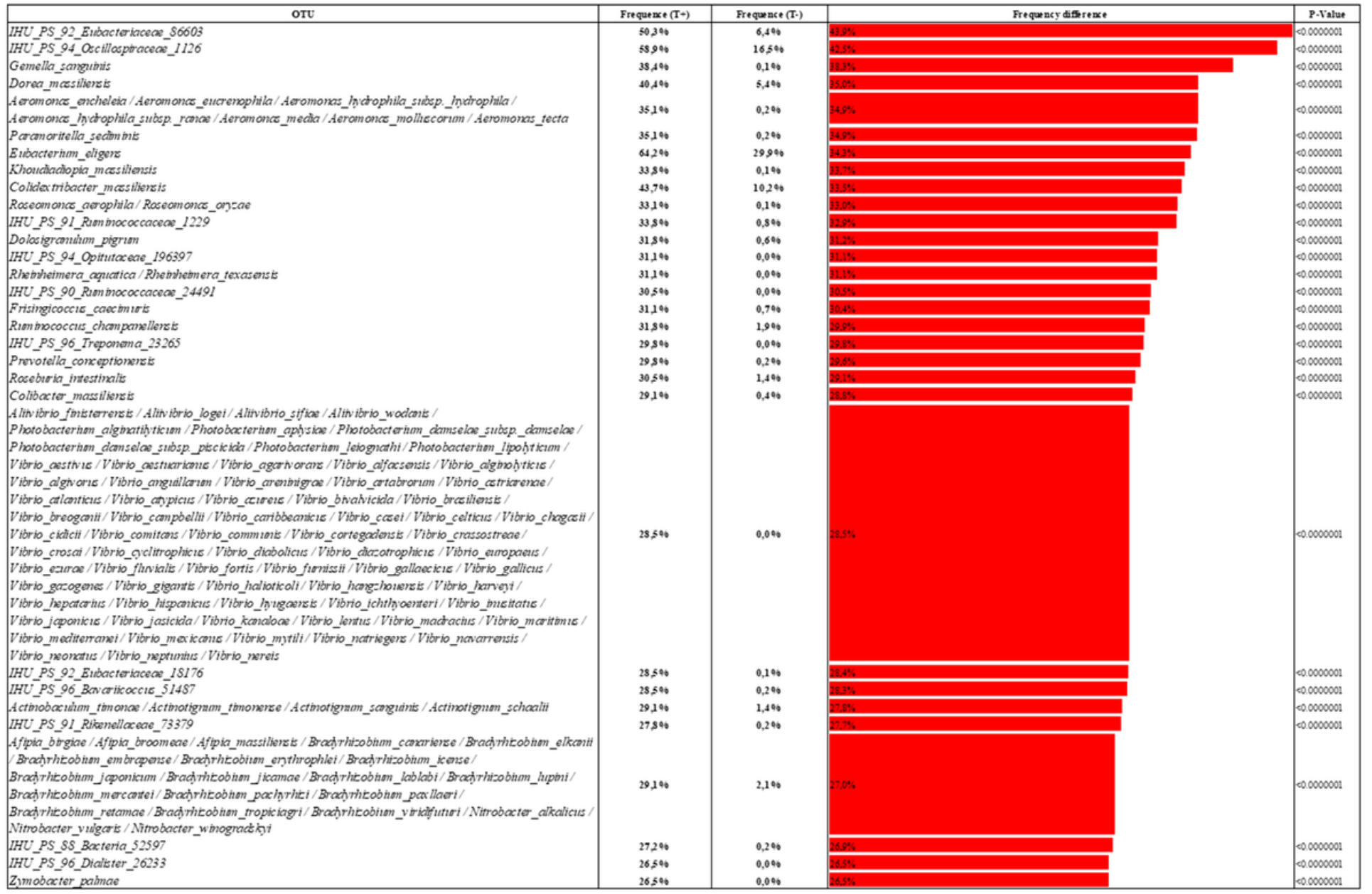

\section{Figure 6}

Most frequent OTUs associated to the prevalence in Treponema spp. The frequency difference of each OTU was calculated between the metagenomes prevalent in Treponema spp. ( $T+$ group) and the metagenomes in which Treponema spp. were not detected (T- group). The 30 most frequent OTUs in the $T+$ group are represented in this figure with their respective frequency in the $T+$ and $T$ - metagenomes as well as the associated $p$ value. 


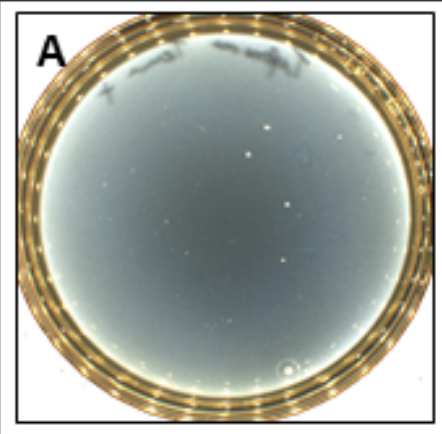

Control 1:T denticolain agar plate

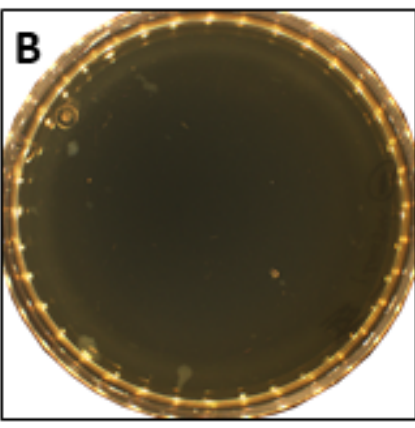

Control 2: Sterile agar plate

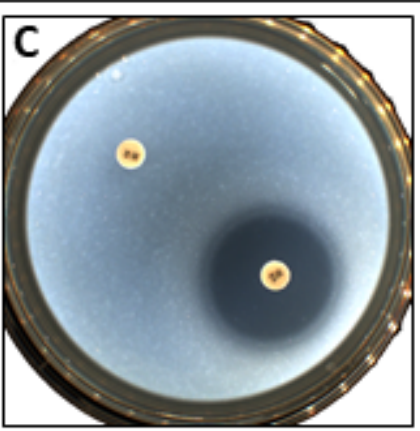

Control 3: $T$. denticolaVs vancomycin and fosfonycin

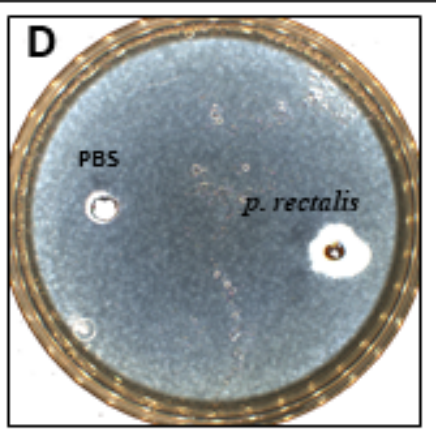

Control 4: $T$. denticolaVs prevotella rectalis

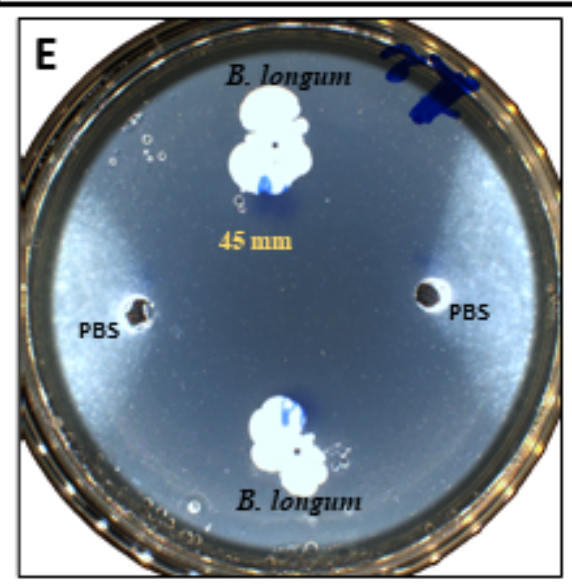

T. denticola Vs B. longum

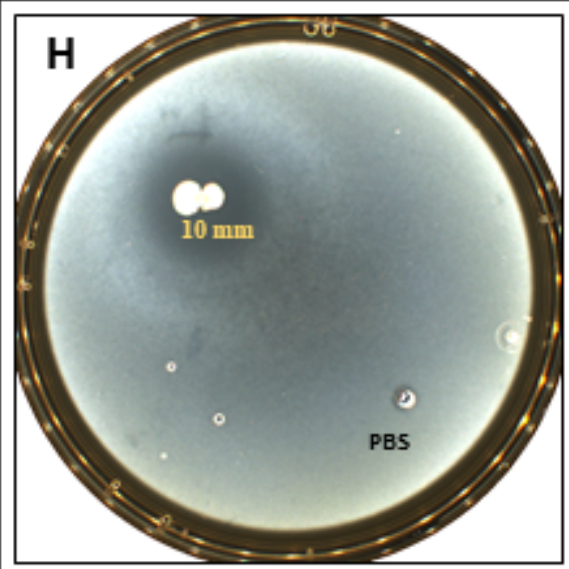

T. denticola Vs B. ovatus

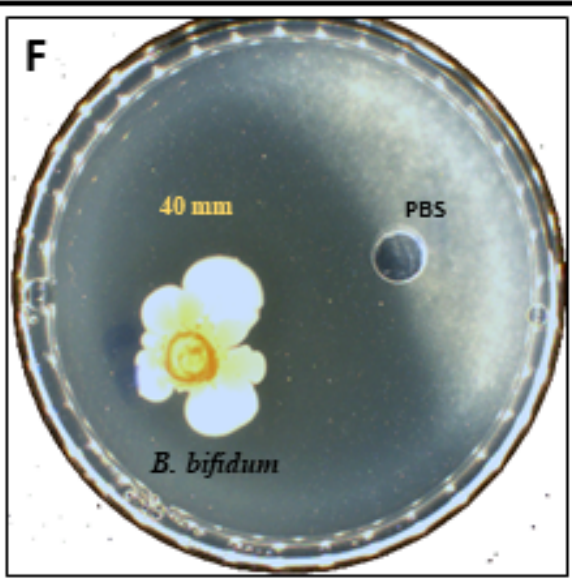

T. denticola Vs B. bifidum

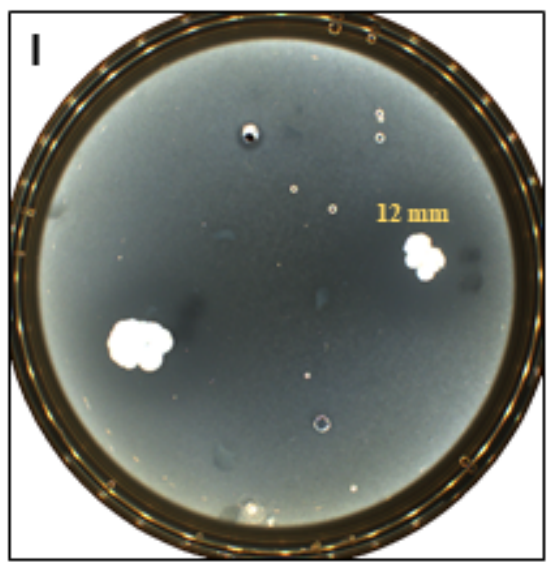

T. denticola V's B. uniformis

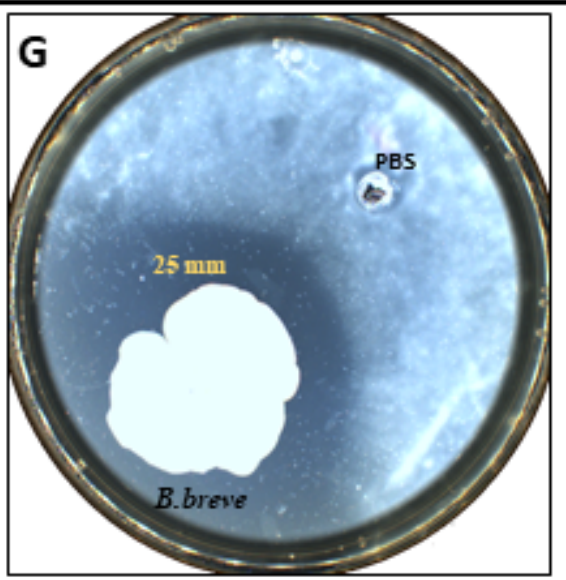

T. denticola Vs B.breve

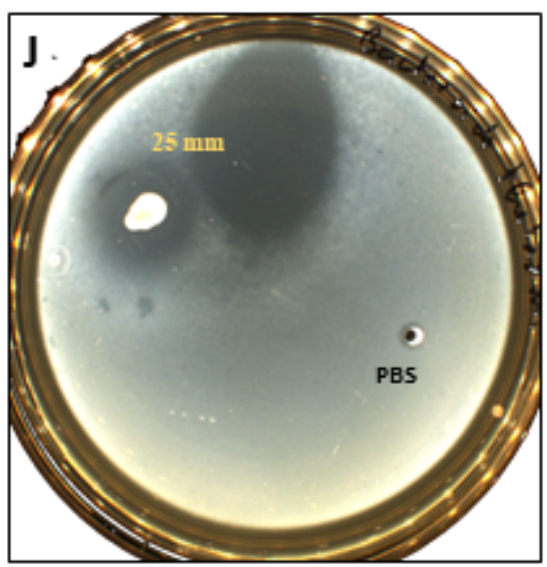

T. denticola Vs B. thetaiotaomicron

\section{Figure 7}

Growth inhibition test between Treponema denticola (CSURP 7640) and predicted antagonists. Examples of the controls performed in these experiments are represented: (A) control 1: growth control, (B) control 2: incubated sterile agar, (C) control 3: positive (vancomycin) and negative (fosfomycin) controls and (D) control 4: T. denticola vs Prevotella rectalis (CSUR P4334). Growth inhibition of T. denticola was obtained with Bifidobacterium ( $\mathrm{E}, \mathrm{F}$ and $\mathrm{G})$ and Bacteroides $(\mathrm{H}, \mathrm{I}$ and $\mathrm{J})$. 


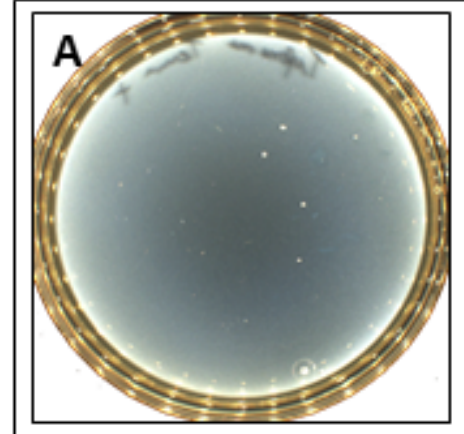

Control 1:T denticolain agar plate

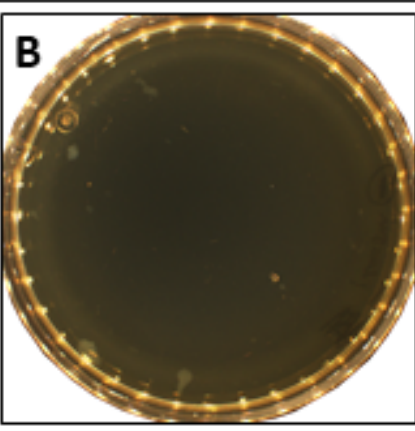

Control 2: Sterile agar plate

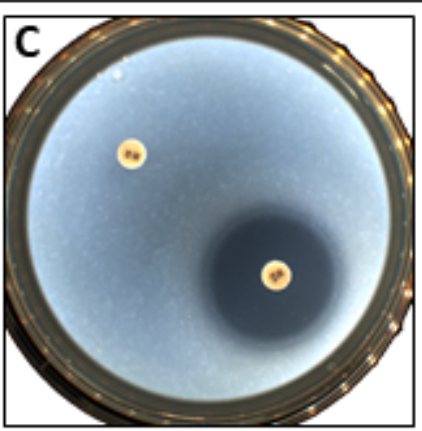

Control 3: $T$. denticolaVs vancomycin and fosfonycin

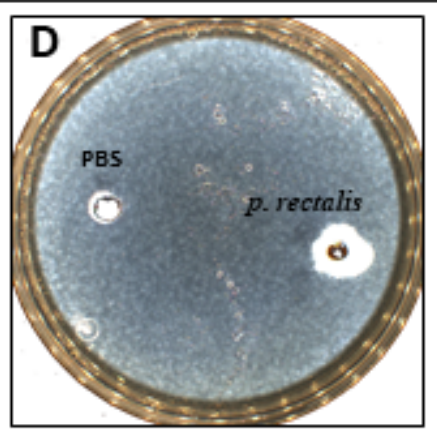

Control 4: $T$. denticolaVs prevotella rectalis

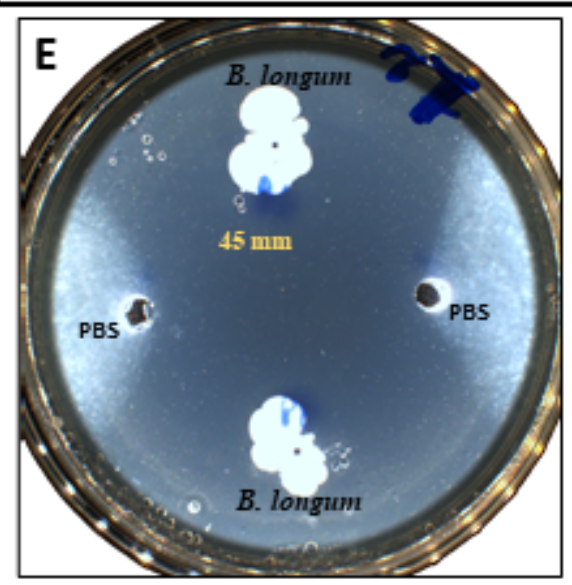

T. denticola Vs B. longum

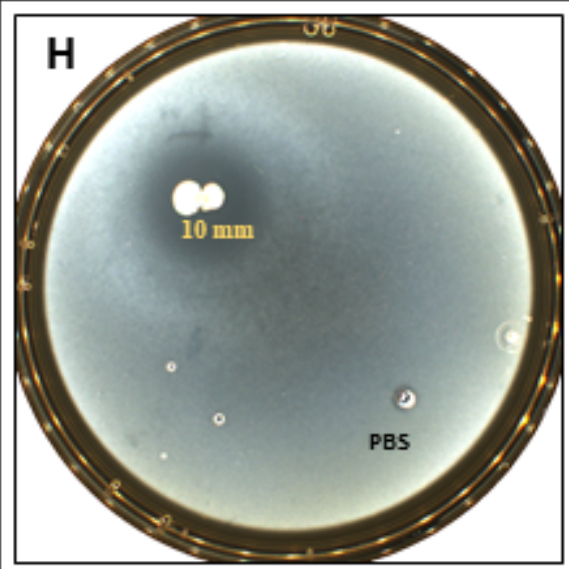

T. denticola Vs B. ovatus

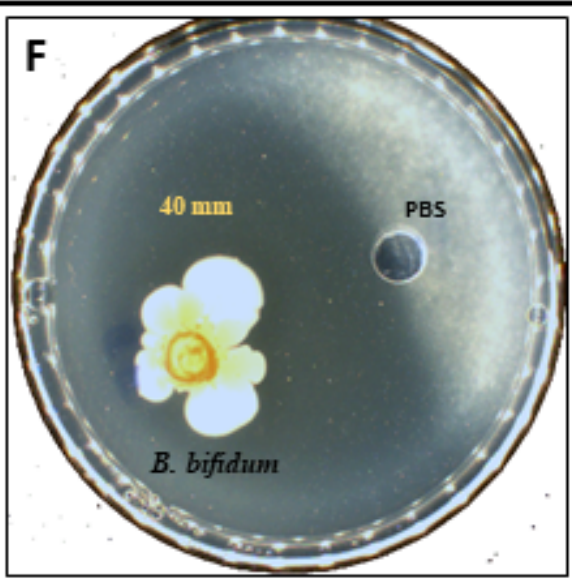

T. denticola Vs B. bifidum

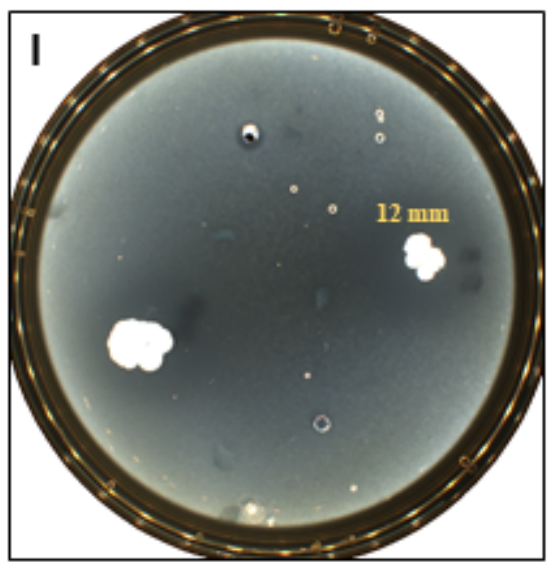

T. denticola V's B. uniformis

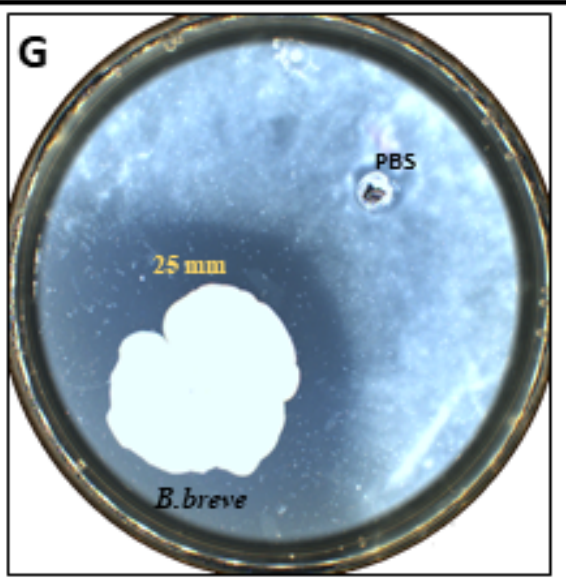

T. denticola Vs B.breve

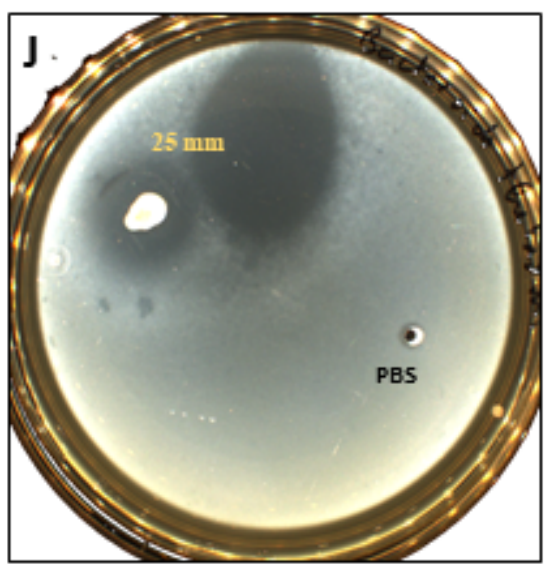

T. denticola Vs B. thetaiotaomicron

\section{Figure 7}

Growth inhibition test between Treponema denticola (CSURP 7640) and predicted antagonists. Examples of the controls performed in these experiments are represented: (A) control 1: growth control, (B) control 2: incubated sterile agar, (C) control 3: positive (vancomycin) and negative (fosfomycin) controls and (D) control 4: T. denticola vs Prevotella rectalis (CSUR P4334). Growth inhibition of T. denticola was obtained with Bifidobacterium ( $\mathrm{E}, \mathrm{F}$ and $\mathrm{G})$ and Bacteroides $(\mathrm{H}, \mathrm{I}$ and $\mathrm{J})$.

\section{Supplementary Files}


This is a list of supplementary files associated with this preprint. Click to download.

- SupplementaryFile.zip

- SupplementaryFile.zip 